\title{
Lattice Points on the Fermat Factorization Method
}

\author{
Regis Freguin Babindamana $\mathbb{D}$, Gilda Rech Bansimba $\mathbb{D}$, and Basile Guy Richard Bossoto
}

Université Marien Ngouabi, Faculté des Sciences et Techniques, BP: 69, Brazzaville, Congo

Correspondence should be addressed to Regis Freguin Babindamana; regis.babindamana@umng.cg

Received 5 May 2021; Revised 17 August 2021; Accepted 6 December 2021; Published 28 January 2022

Academic Editor: Bibhas Ranjan Majhi

Copyright (C) 2022 Regis Freguin Babindamana et al. This is an open access article distributed under the Creative Commons Attribution License, which permits unrestricted use, distribution, and reproduction in any medium, provided the original work is properly cited.

\begin{abstract}
In this paper, we study algebraic properties of lattice points of the arc on the conics $x^{2}-d y^{2}=N$ especially for $d=1$, which is the Fermat factorization equation that is the main idea of many important factorization methods like the quadratic field sieve, using arithmetical results of a particular hyperbola parametrization. As a result, we present a generalization of the forms, the cardinal, and the distribution of its lattice points over the integers. In particular, we prove that if $(N-6) \equiv 0 \bmod 4$, Fermat's method fails. Otherwise, in terms of cardinality, it has, respectively, 4, 8, $2(\alpha+1),\left(1-\delta_{2 p_{i}}\right) 2^{n+1}$, and $2 \prod_{i=1}^{n}\left(\alpha_{i}+1\right)$ lattice pointts if $N$ is an odd prime, $N=N_{a} \times N_{b}$ with $N_{a}$ and $N_{b}$ being odd primes, $N=N_{a}^{\alpha}$ with $N_{a}$ being prime, $N=\prod_{i=1}^{n} p_{i}$ with $p_{i}$ being distinct primes, and $N=\prod_{i=1}^{n} N_{i}^{\alpha_{i}}$ with $N_{i}$ being odd primes. These results are important since they provide further arithmetical understanding and information on the integer solutions revealing factors of $N$. These results could be particularly investigated for the purpose of improving the underlying integer factorization methods.
\end{abstract}

\section{Introduction}

Diophantine equations have been for many decades a very important subject of research in number theory, and lattice points on curves have been studied in the literature particularly by Gauss, and bounds on arcs of conics have also been studied since then (see [1-6]). However, the necessity of representing an integer as difference of two squares, i.e., for a given $N \in \mathbb{Z}$, finding nontrivial couples $(x, y) \in \mathbb{Z}^{2}$ such that $x^{2}-y^{2}=N$, appears in the literature as the main idea of many factorization methods (see $[7,8]$ ) as suggested by Fermat (see $[9,10]$ ). While being not hard to observe, its lattice points are easily computable if one knows the factorization of $N$, and in contrast, this gets exponentially harder when it comes to special cases of $N$, mainly when $N=\prod_{i=1}^{j} p_{i}$, where $p_{i}$ are large primes, in which case this problem becomes equivalent to factoring the parameter $N$.

For this reason, one of fundamental research problems on conics is to find integral solutions of particular hyperbola parametrizations mainly $x^{2}-y^{2}=N$ over the integers, particularly when $N$ is a large semiprime, in which case if a computationally efficient algorithm is found, cryptosystems like RSA [11] would no longer be secured.
Reviewing the literature, some results on various hyperbola parametrizations and their applications have been studied. Particularly in [1], Javier and Jorge used ideals in quadratic field $\mathbb{Q}(\sqrt{d})$ to find an upper bound for the number of lattice points on Pell's equation $x^{2}-d y^{2}=N$, while in [12], Jin et al. used results from the forms of integral solutions of the hyperbola $b x^{2}-a b x y+a y^{2}=k$ to solve the same equation where $d$ is of the form $p^{2}-q>0$. In [13], the author studied a special case of hyperbola and presented the forms of its integral points over $\mathbb{Z}$, and in [14], Yeonok investigated some behaviors of integral points on the hyperbola $b x^{2}-a b x y+a y^{2}=-b k\left(k \in \mathbb{Z}_{>0}\right)$ to the generalizations of Binet formula and Catalan's identity, while in [15], the authors gave an application of group law on affine conics to cryptography. Still, in the previous works, algebraic properties and distribution of lattice points and cardinalities on Fermat's equation are not presented. More recently, in [16], Gilda et al. investigated algebraic and arithmetical properties on the group structure $\mathscr{B}_{N}(x, y)=$ $\left\{(x, y) \in \mathbb{Q} \times \mathbb{Q} / y^{2}=x^{2}-4 N x\right\}$, mainly isomorphisms, integral solutions, and a description of a factorization method with no generalization to the Fermat factorization equation. 
In this paper, we use the hyperbola parametrization introduced in [16] to study algebraic properties of lattice points and their distribution for Fermat's factorization equation for which we find exact upper and lower bounds and we present the forms and cardinalities with a generalization of results for most of special cases of $N$, using results from the particular hyperbola parametrization.

The article is organized as follows:

(i) In Section 1, we give an introduction

(ii) In Section 2, we present the particular hyperbola parametrization and related arithmetical results

(iii) In Section 3, we present the application of the hyperbola parametrization to the study of lattice points on the Fermat equation

(iv) In Section 4, In Section 4, we do a discussion on the likelihood of finding solutions to the Fermat factorization equation

(v) In Section 5, we finally conclude

Here is a list of the commonly used nomenclature in this paper:

$\mathscr{B}_{N}(x, y)_{\mid \mathbb{Q}}=\left\{(x, y) \in \mathbb{Q} \times \mathbb{Q} / y^{2}=x^{2}-4 N x\right\}:$ algebraic set of all rational points on $\mathscr{B}_{N}(x, y)$.

$\mathscr{B}_{N}(x, y)_{\mid \mathbb{Z}}=\left\{(x, y) \in \mathbb{Z} \times \mathbb{Z} / y^{2}=x^{2}-4 N x\right\}$ : algebraic set of all integral points on $\mathscr{B}_{N}(x, y)$.

$\mathscr{B}_{N}(x, y)_{\mid x \geq 4 N}=\left\{(x, y) \in \mathbb{Z}_{\geq 4 N} \times \mathbb{Z}_{\geq 0} / y^{2}=x^{2}-4 N x\right\}:$ algebraic set of integral points on $\mathscr{B}_{N}(x, y)$ whose $x$-coordinates are greater or equal to $4 N$.

$\chi_{N_{a} \rightarrow N_{b}}$ : an injective homomorphism from $\mathscr{B}_{N_{a}}(x, y)$ to $\mathscr{B}_{N_{b}}(x, y)$.

$\operatorname{Card}\left(\mathscr{B}_{N}(x, y)\right)$ : the cardinal of $\mathscr{B}_{N}(x, y)$.

$\operatorname{Div}\left(N_{a}\right)$ : set of divisors of $N_{a}$.

$\pi_{p}(n)$ : the set of all prime divisors of $n$.

$H_{N}=\left\{(x, y) \in \mathbb{Z}^{2} / x^{2}-y^{2}=N\right\}$ : the Fermat factorization equation.

$\delta_{i j}$ : the Kronecker symbol.

\section{BN Hyperbola Parametrization}

A conic is an algebraic set satisfying an equation of the form $\alpha_{1} x^{2}+2 \alpha_{2} x y+\alpha_{2} y^{2}+2 \alpha_{3} x+2 \alpha_{4} y+\alpha_{5}=0,\left(\alpha_{1}, \alpha_{2}, \alpha_{3}, \alpha_{4}\right.$, $\left.\alpha_{5}\right) \in \mathbb{R}^{5}$ where $\left(\alpha_{1}, \alpha_{2}, \alpha_{3}\right) \neq(0,0,0)$. Setting $\mathscr{B}_{N}$ the parametrization defined by $(x, y) \in \mathbb{Q} \times \mathbb{Q} / y^{2}=x^{2}-4 N x$, in the projective space $\mathbb{P}^{2}(\mathbb{Q})$, we have $\mathscr{B}_{n}(X, Y, Z)=$ $\left\{(X: Y: Z) \in \mathbb{P}^{2}(\mathbb{Q}) /\left(Y^{2} / Z^{2}\right)=\left(X^{2} / Z^{2}\right)-4 N(X / Z)\right\} \Leftrightarrow$ $\mathscr{B}_{N}(X, Y, Z)=\left\{(X: Y: Z) \in\left(\mathbb{P}^{2}(\mathbb{Q}) / Y^{2}\right)=X^{2}-4 N X Z\right\}$.

At infinity, setting $Z=0$ and considering $X, Y>0$, we obtain $Y^{2}=X^{2}, \quad$ and the equivalence class $(X: Y: Z) \sim(X: X: 0) \sim X(1: 1: 0)$, and hence one of the points at infinity is $P_{\infty}=(1: 1: 0)$.

From now on, $\forall N \in \mathbb{Z}_{>0}, \mathscr{B}_{N}(x, y)$ denotes $\mathscr{B}_{N}(x, y)$ over the field $\mathrm{Q} \mathscr{B}_{N}(x, y)_{\left.\right|_{x>4 N}}$ denotes $\mathscr{B}_{N}(x, y)$ over $\mathbb{Z}_{\geq 4 N} \times \mathbb{Z}_{\geq 0}$ and $\mathscr{B}_{N}(x, y)_{\left.\right|_{\mathbb{Z}}}$ denotes $\mathscr{B}_{N}(x, y)$ over the integers, i.e., $\mathscr{B}_{N}(x, y)=\left\{(x, y) \in \mathbb{Q} \times \mathbb{Q} / y^{2}=x^{2}-4 N x\right\}$; and $\mathscr{B}_{N}(x, y)_{\left.\right|_{x>4 N}}=\left\{(x, y) \in \mathbb{Z}_{\geq 4 N} \times \mathbb{Z}_{\geq 0} / y^{2}=x^{2}-4 N x\right\}$ and $\mathscr{B}_{N}(x, y)_{\left.\right|_{\mathbb{Z}}}=\left\{(x, y) \in \mathbb{Z}^{2} / y^{2}=x^{2}-4 N x\right\}$.

Proposition 1. Consider the application

$+: \mathscr{B}_{N}(x, y) \times \mathscr{B}_{N}(x, y) \longrightarrow \mathscr{B}_{N}(x, y)(P, Q) \mapsto P+Q$.

$P+Q \quad$ is defined $\forall P=\left(x_{p}, y_{p}\right), \quad Q=\left(x_{q}, y_{q}\right) \in$ $\mathscr{B}_{N}(x, y)$, by

$\left\{\begin{array}{l}x_{p+q}=\frac{1}{2 N}\left[\left(x_{p}-2 N\right)\left(x_{q}-2 N\right)+y_{p} y_{q}\right]+2 N, \\ y_{p+q}=\frac{1}{2 N}\left[y_{p}\left(x_{q}-2 N\right)+y_{q}\left(x_{p}-2 N\right)\right],\end{array}\right.$

$\left\{\begin{array}{l}x_{2 p}=\frac{1}{2 N}\left[\left(x_{p}-2 N\right)^{2}+y_{p}^{2}\right]+2 N, \\ y_{2 p}=\frac{1}{N}\left(y_{p}\left(x_{p}-2 N\right)\right) .\end{array}\right.$

Then, $\left(\mathscr{B}_{N}(x, y),+\right)$ is an abelian group with neutral element $\mathcal{O}=(4 N, 0)$.

Proof. Let us consider the affine space $\mathbb{Q}^{2}(x, y)$.

$\mathscr{B}_{N}$ is a hyperbola of equation $X Y=1$, where $X=(x-y-2 N / 2 N), Y=(x+y-2 N / 2 N)$, i.e., $(x-2 N /$ $2 N)^{2}-(y / 2 N)^{2}=1$, with $N \in \mathbb{Z}_{>0}$. Set

$$
\begin{aligned}
& \left\{\begin{array} { l } 
{ x - 2 N = 2 N \operatorname { c o s h } ( t ) } \\
{ y = 2 N \operatorname { s i n h } ( t ) }
\end{array} \Rightarrow \left\{\begin{array}{l}
\frac{x}{2 N}-1=\cosh (t) \\
\frac{y}{2 N}=\sinh (t)
\end{array} \quad \sin c e \cosh ^{2}(t)-\sinh ^{2}(t)=1,\right.\right.
\end{aligned}
$$


Given $\quad$ two
$\left(x_{q}, y_{q}\right) \in \mathscr{B}_{N}(x, y)$,

$$
\begin{aligned}
& \operatorname{Set}\left\{\begin{array}{l}
X_{1}=\frac{x_{p}}{2 N}-1=\cosh \left(t_{1}\right) \\
Y_{1}=\frac{y_{p}}{2 N}=\sinh \left(t_{1}\right)
\end{array}\right. \\
& \left\{\begin{array} { l } 
{ X _ { 2 } = \frac { x _ { q } } { 2 N } - 1 = \operatorname { c o s h } ( t _ { 2 } ) , } \\
{ Y _ { 2 } = \frac { y _ { q } } { 2 N } = \operatorname { s i n h } ( t _ { 2 } ) , }
\end{array} \text { We then have } \left\{\begin{array}{l}
\cosh \left(t_{1}+t_{2}\right)=X_{1} X_{2}+Y_{1} Y_{2}, \\
\sinh \left(t_{1}+t_{2}\right)=Y_{1} X_{2}+X_{1} Y_{2} .
\end{array}\right.\right.
\end{aligned}
$$

We easily verify that $\left(X_{1} X_{2}+Y_{1} Y_{2}\right)^{2}-\left(Y_{1} X_{2}+\right.$ $\left.X_{1} Y_{2}\right)^{2}=1$.

$$
\begin{aligned}
& \left\{\begin{array} { l } 
{ \frac { x _ { p + q } } { 2 N } - 1 = X _ { 1 } X _ { 2 } + Y _ { 1 } Y _ { 2 } } \\
{ \frac { y _ { p + q } } { 2 N } = Y _ { 1 } X _ { 2 } + X _ { 1 } Y _ { 2 } }
\end{array} \Rightarrow \left\{\begin{array} { l } 
{ \frac { x _ { p + q } } { 2 N } = X _ { 1 } X _ { 2 } + Y _ { 1 } Y _ { 2 } + 1 } \\
{ \frac { y _ { p + q } } { 2 N } = Y _ { 1 } X _ { 2 } + X _ { 1 } Y _ { 2 } }
\end{array} \Rightarrow \left\{\begin{array}{l}
x_{p+q}=2 N\left(X_{1} X_{2}+Y_{1} Y_{2}+1\right) \\
y_{p+q}=2 N\left(Y_{1} X_{2}+X_{1} Y_{2}\right)
\end{array},\right.\right.\right. \\
& X_{1} X_{2}+Y_{1} Y_{2}+1=\left(\frac{x_{p}}{2 N}-1\right)\left(\frac{x_{q}}{2 N}-1\right)+\left(\frac{y_{p}}{2 N}\right)\left(\frac{y_{q}}{2 N}\right)+1=\frac{\left(x_{p}-2 N\right)\left(x_{q}-2 N\right)+y_{p} y_{q}}{4 N^{2}}+1 \text {, } \\
& \Rightarrow x_{p+q}=2 N\left(X_{1} X_{2}+Y_{1} Y_{2}+1\right)=\frac{1}{2 N}\left[\left(x_{p}-2 N\right)\left(x_{q}-2 N\right)+y_{p} y_{q}\right]+2 N \\
& Y_{1} X_{2}+X_{1} Y_{2}=\left(\frac{y_{p}}{2 N}\right)\left(\frac{x_{q}}{2 N}-1\right)+\left(\frac{y_{q}}{2 N}\right)\left(\frac{x_{p}}{2 N}-1\right)=\frac{1}{4 N^{2}}\left[y_{p}\left(x_{q}-2 N\right)+y_{q}\left(x_{p}-2 N\right)\right] \\
& \Rightarrow y_{p+q}=2 N\left(Y_{1} X_{2}+X_{1} Y_{2}\right)=\frac{1}{2 N}\left[y_{p}\left(x_{q}-2 N\right)+y_{q}\left(x_{p}-2 N\right)\right] \text {. }
\end{aligned}
$$

We have both

$$
\begin{aligned}
& \left\{\begin{array}{l}
x_{p+q}=\frac{1}{2 N}\left[\left(x_{p}-2 N\right)\left(x_{q}-2 N\right)+y_{p} y_{q}\right]+2 N, \\
y_{p+q}=\frac{1}{2 N}\left[y_{p}\left(x_{q}-2 N\right)+y_{q}\left(x_{p}-2 N\right)\right],
\end{array}\right. \\
& \left\{\begin{array}{l}
x_{2 p}=\frac{1}{2 N}\left[\left(x_{p}-2 N\right)^{2}+y_{p}^{2}\right]+2 N, \\
y_{2 p}=\frac{1}{N}\left(y_{p}\left(x_{p}-2 N\right)\right) .
\end{array}\right.
\end{aligned}
$$

We denote + as the above defined additive law. This addition law is strongly unified since point doubling does exist and is well defined.
Now let us consider the application

$$
\begin{aligned}
+ & : \mathscr{B}_{N}(x, y) \times \mathscr{B}_{N}(x, y) \longrightarrow \mathscr{B}_{N}(x, y) \\
& (P, Q) \mapsto P+Q .
\end{aligned}
$$

This application is an internal composition law since $P+Q \in \mathscr{B}_{N}(x, y),\left(y_{p+q}^{2}=x_{p+q}^{2}-4 N x_{p+q}\right)$.

(i) Associativity: $\quad$ given 3 points $P=\left(x_{p}, y_{p}\right), Q=\left(x_{q}, y_{q}\right), T=\left(x_{t}, y_{t}\right) \in \mathscr{B}_{N}(x, y)$, here we show that $(P+Q)+T=P+(Q+T)$.

Note that this can be shown either geometrically or analytically, but here we give just the analytic proof.

Consider $(P+Q)+T$. 


$$
\begin{aligned}
& (P+Q)+T=\left\{\begin{array}{l}
x_{(p+q)+t}=\frac{1}{2 N}\left[\left(x_{(p+q)}-2 N\right)\left(x_{t}-2 N\right)+y_{(p+q)} y_{t}\right]+2 N \\
y_{(p+q)+t}=\frac{1}{2 N}\left[y_{(p+q)}\left(x_{t}-2 N\right)+y_{t}\left(x_{(p+q)}-2 N\right)\right]
\end{array}\right. \\
& =\left\{\begin{array}{l}
x_{(p+q)+t}=\frac{1}{2 N}\left[\left(\frac{1}{2 N}\left[\left(x_{p}-2 N\right)\left(x_{q}-2 N\right)+y_{p} y_{q}\right]+2 N-2 N\right)\left(x_{t}-2 N\right)+\left(\frac{1}{2 N}\left[y_{p}\left(x_{q}-2 N\right)+y_{q}\left(x_{p}-2 N\right)\right]\right) y_{t}\right]+2 N \\
y_{(p+q)+t}=\frac{1}{2 N}\left[\left(\frac{1}{2 N}\left[y_{p}\left(x_{q}-2 N\right)+y_{q}\left(x_{p}-2 N\right)\right]\right)\left(x_{t}-2 N\right)+y_{t}\left(\frac{1}{2 N}\left[\left(x_{p}-2 N\right)\left(x_{q}-2 N\right)+y_{p} y_{q}\right]+2 N-2 N\right)\right]
\end{array}\right. \\
& =\left\{\begin{array}{l}
x_{(p+q)+t}=\frac{1}{2 N}\left[\left(\frac{1}{2 N}\left[\left(x_{p}-2 N\right)\left(x_{q}-2 N\right)+y_{p} y_{q}\right]\right)\left(x_{t}-2 N\right)+\left(\frac{1}{2 N}\left[y_{p}\left(x_{q}-2 N\right)+y_{q}\left(x_{p}-2 N\right)\right]\right) y_{t}\right]+2 N \\
y_{(p+q)+t}=\frac{1}{2 N}\left[\left(\frac{1}{2 N}\left[y_{p}\left(x_{q}-2 N\right)+y_{q}\left(x_{p}-2 N\right)\right]\right)\left(x_{t}-2 N\right)+y_{t}\left(\frac{1}{2 N}\left[\left(x_{p}-2 N\right)\left(x_{q}-2 N\right)+y_{p} y_{q}\right]\right)\right] .
\end{array}\right.
\end{aligned}
$$

$(9)=\left\{\begin{array}{l}x_{(p+q)+t}=\frac{1}{2 N}\left[\frac{1}{2 N}\left(x_{p}-2 N\right)\left(x_{q}-2 N\right)\left(x_{t}-2 N\right)+\frac{1}{2 N} y_{p} y_{q}\left(x_{t}-2 N\right)+\frac{1}{2 N} y_{p} y_{t}\left(x_{q}-2 N\right)+\frac{1}{2 N} y_{q} y_{t}\left(x_{p}-2 N\right)\right]+2 N, \\ y_{(p+q)+t}=\frac{1}{2 N}\left[\frac{1}{2 N} y_{p}\left(x_{q}-2 N\right)\left(x_{t}-2 N\right)+\frac{1}{2 N} y_{q}\left(x_{p}-2 N\right)\left(x_{t}-2 N\right)+\frac{1}{2 N} y_{t}\left(x_{p}-2 N\right)\left(x_{q}-2 N\right)+\frac{1}{2 N} y_{p} y_{q} y_{t}\right]\end{array}\right.$

Secondly we consider $P+(Q+T)=\left\{\begin{array}{l}x_{p+(q+t)}=\frac{1}{2 N}\left[\left(x_{p}-2 N\right)\left(x_{(q+t)}-2 N\right)+y_{p} y_{(q+t)}\right]+2 N \\ y_{p+(q+t)}=\frac{1}{2 N}\left[y_{p}\left(x_{(q+t)}-2 N\right)+y_{(q+t)}\left(x_{p}-2 N\right)\right]\end{array}\right.$

$$
\begin{aligned}
& =\left\{\begin{array}{l}
x_{p+(q+t)}=\frac{1}{2 N}\left[\left(x_{p}-2 N\right)\left(\frac{1}{2 N}\left[\left(x_{q}-2 N\right)\left(x_{t}-2 N\right)+y_{q} y_{t}\right]+2 N-2 N\right)+y_{p} \frac{1}{2 N}\left[y_{q}\left(x_{t}-2 N\right)+y_{t}\left(x_{q}-2 N\right)\right]\right]+2 N \\
y_{p+(q+t)}=\frac{1}{2 N}\left[y_{p}\left(\frac{1}{2 N}\left[\left(x_{q}-2 N\right)\left(x_{t}-2 N\right)+y_{q} y_{t}\right]+2 N-2 N\right)+\frac{1}{2 N}\left[y_{q}\left(x_{t}-2 N\right)+y_{t}\left(x_{q}-2 N\right)\right]\left(x_{p}-2 N\right)\right]
\end{array}\right. \\
& =\left\{\begin{array}{l}
x_{p+(q+t)}=\frac{1}{2 N}\left[\left(x_{p}-2 N\right)\left(\frac{1}{2 N}\left[\left(x_{q}-2 N\right)\left(x_{t}-2 N\right)+y_{q} y_{t}\right]\right)+y_{p} \frac{1}{2 N}\left[y_{q}\left(x_{t}-2 N\right)+y_{t}\left(x_{q}-2 N\right)\right]\right]+2 N \\
y_{p+(q+t)}=\frac{1}{2 N}\left[y_{p}\left(\frac{1}{2 N}\left[\left(x_{q}-2 N\right)\left(x_{t}-2 N\right)+y_{q} y_{t}\right]\right)+\frac{1}{2 N}\left[y_{q}\left(x_{t}-2 N\right)+y_{t}\left(x_{q}-2 N\right)\right]\left(x_{p}-2 N\right)\right],
\end{array}\right.
\end{aligned}
$$

$(11)=\left\{\begin{array}{l}x_{p+(q+t)}=\frac{1}{2 N}\left[\frac{1}{2 N}\left(x_{p}-2 N\right)\left(x_{q}-2 N\right)\left(x_{t}-2 N\right)+\frac{1}{2 N} y_{q} y_{t}\left(x_{p}-2 N\right)+\frac{1}{2 N} y_{p} y_{q}\left(x_{t}-2 N\right)+\frac{1}{2 N} y_{p} y_{t}\left(x_{q}-2 N\right)\right]+2 N \\ y_{p+(q+t)}=\frac{1}{2 N}\left[\frac{1}{2 N} y_{p}\left(x_{q}-2 N\right)\left(x_{t}-2 N\right)+\frac{1}{2 N} y_{p} y_{q} y_{t}+\frac{1}{2 N} y_{q}\left(x_{t}-2 N\right)\left(x_{p}-2 N\right)+\frac{1}{2 N} y_{t}\left(x_{q}-2 N\right)\left(x_{p}-2 N\right)\right] .\end{array}\right.$

We clearly see by identification that (9) $=(11) \Rightarrow(P+Q)+T=P+(Q+T)$. 
(i) Neutral element: $\mathcal{O}=(4 N, 0)$. It is obvious to see that $\mathcal{O}=(4 N, 0) \in \mathscr{B}_{N}(x, y)$. Given any point $P=\left(x_{p}, y_{p}\right) \in \mathscr{B}(x, y)$, from (3), we have

$$
\begin{aligned}
& P+\mathcal{O}=\left\{\begin{array}{l}
x_{p+\mathcal{O}}=\frac{1}{2 n}\left[\left(x_{p}-2 N\right)(4 N-2 N)+y_{p} \times 0\right]+2 N=\frac{1}{2 N}\left[2 N\left(x_{p}-2 N\right)\right]+2 N=x_{p} \\
y_{p+\mathcal{O}}=\frac{1}{2 N}\left[y_{p}(4 N-2 N)+0 \times\left(x_{p}-2 N\right)\right]=\frac{1}{2 N}\left[2 N y_{p}\right]=y_{p},
\end{array}\right. \\
& \mathcal{O}+P=\left\{\begin{array}{l}
x_{\mathscr{O}+p}=\frac{1}{2 N}\left[(4 N-2 N)\left(x_{p}-2 N\right)+0 \times y_{p}\right]+2 N=\frac{1}{2 N}\left[2 N\left(x_{p}-2 N\right)\right]+2 N=x_{p} \\
y_{\mathcal{O}+p}=\frac{1}{2 N}\left[0 \times\left(x_{p}-2 N\right)+y_{p}(4 N-2 N)\right]=\frac{1}{2 N}\left[2 N y_{p}\right]=y_{p} .
\end{array}\right.
\end{aligned}
$$

Hence, $\forall P \in \mathscr{B}_{N}(x, y), P+\mathcal{O}=\mathcal{O}+P=P$.

(ii) Symmetric element: $\forall P=\left(x_{p}, y_{p}\right) \in \mathscr{B}(x, y), P^{\prime}=$ $\left(x_{p},-y_{p}\right)$ is the symmetric element of P. It is obvious to see that $\left(x_{p},-y_{p}\right) \in \mathscr{B}_{N}(x, y)$. Then, we have

$$
\begin{aligned}
& P+P^{\prime}=\left\{\begin{array}{l}
x_{p+p^{\prime}}=\frac{1}{2 N}\left[\left(x_{p}-2 N\right)^{2}-y_{p}^{2}\right]+2 N=\frac{1}{2 N}\left[x_{p}^{2}-4 N x_{p}+4 N^{2}-y_{p}^{2}\right]+2 N=\frac{1}{2 N}\left[4 N^{2}\right]+2 N=4 N \\
y_{p+p^{\prime}}=\frac{1}{2 N}\left[y_{p}\left(x_{p}-2 N\right)-y_{p}\left(x_{p}-2 N\right)\right]=\frac{1}{2 N}[0]=0,
\end{array}\right. \\
& P^{\prime}+P=\left\{\begin{array}{l}
x_{p^{\prime}+p}=\frac{1}{2 N}\left[\left(x_{p}-2 N\right)^{2}-y_{p}^{2}\right]+2 N=\frac{1}{2 N}\left[x_{p}^{2}-4 N x_{p}+4 N^{2}-y_{p}^{2}\right]+2 N=\frac{1}{2 N}\left[4 N^{2}\right]+2 N=4 N \\
y_{p^{\prime}+p}=\frac{1}{2 N}\left[-y_{p}\left(x_{p}-2 N\right)+y_{p}\left(x_{p}-2 N\right)\right]=\frac{1}{2 N}[0]=0 .
\end{array}\right.
\end{aligned}
$$

Hence, $\forall P=\left(x_{p}, y_{p}\right), P^{\prime}=\left(x_{p},-y_{p}\right) \in \mathscr{B}_{N}(x, y)$,

$P+P^{\prime}=P^{\prime}+P=\mathscr{O}$.

(iii) Commutativity:

$$
P+Q=\left\{\begin{array}{l}
x_{p+q}=\frac{1}{2 N}\left[\left(x_{p}-2 N\right)\left(x_{q}-2 N\right)+y_{p} y_{q}\right]+2 N=\frac{1}{2 N}\left[\left(x_{q}-2 N\right)\left(x_{p}-2 N\right)+y_{q} y_{p}\right]+2 N=x_{q+p}, \\
y_{p+q}=\frac{1}{2 N}\left[y_{p}\left(x_{q}-2 N\right)+y_{q}\left(x_{p}-2 N\right)\right]=\frac{1}{2 N}\left[y_{q}\left(x_{p}-2 N\right)+y_{p}\left(x_{q}-2 N\right)\right]=y_{q+p} .
\end{array}\right.
$$

Hence, $\forall P, Q \in \mathscr{B}_{N}(x, y), P+Q=Q+P$.

Proposition 2. Let $\mathscr{B}_{N_{a}}(x, y)=\left\{(x, y) \in \mathbb{Q} \times \mathbb{Q} / y^{2}=x^{2}-\right.$ $\left.4 N_{a} x\right\}$ and $\mathscr{B}_{N_{b}}(x, y) \stackrel{N_{a}}{=}\left\{(x, y) \in \mathbb{Q} \times \mathbb{Q} / y^{2}=x^{2}-4 N_{b} x\right\}$. Then, the following map:

$\chi: \mathscr{B}_{N_{a}}(x, y) \longrightarrow \mathscr{B}_{N_{b}}(x, y)(x, y) \mapsto\left(x \frac{N_{b}}{N_{a}}, y \frac{N_{b}}{N_{a}}\right)$, defines a group homomorphism.

Proof. $\quad \forall P=\left(x_{p}, y_{p}\right) \in \mathscr{B}_{N_{a}}(x, y), \quad \chi(P)=\chi\left(x_{p}, y_{p}\right)=$ $\left(x_{p}\left(N_{b} / N_{a}\right), y_{p}\left(N_{b} / N_{a}\right)\right) \epsilon^{?} \mathscr{B}_{N_{b}}(x, y) . \quad\left(x_{p}\left(N_{b} / N_{a}\right), y_{p}\right.$ $\left.\left(N_{b} / N_{a}\right)\right) \in \mathscr{B}_{N_{b}}(x, y) \Leftrightarrow\left(y_{p}\left(N_{b} / N_{a}\right)\right)^{2}=\left(x_{p}\left(N_{b} / N_{a}\right)\right)^{2}$ $-4 N_{b}\left(x_{p}\left(N_{b} / N_{a}\right)\right)=x_{p}\left(N_{b}^{2} / N_{a}\right)\left(\left(x_{p} / N_{a}\right)-4\right)=N_{b}^{2}\left(x_{p}^{2}\right.$ $\left.-4 N_{a} x_{p}\right) / N_{a}^{2} \Rightarrow\left(y_{p}\left(N_{b} / N_{a}\right)\right)^{2}=N_{b}^{2} y_{p}^{2} / N_{a}^{2}=\left(y_{p}\left(N_{b} /\right.\right.$ $\left.\left.N_{a}\right)\right)^{2}$. Thus, $\left(x_{p}\left(N_{b} / N_{a}\right), y_{p}\left(N_{b} / N_{a}\right)\right) \in \mathscr{B}_{N_{b}}(x, y)$. 
Consider $\mathcal{O}_{\mathscr{B}_{N_{a}}}=\left(4 N_{a}, 0\right)$ and $\mathcal{O}_{\mathscr{B}_{N_{b}}}=\left(4 N_{b}, 0\right)$.

$$
\chi\left(\mathcal{O}_{\mathscr{B}_{N_{a}}}\right)=\chi\left(\left(4 N_{a}, 0\right)\right)=\left(4 N_{a} \frac{N_{b}}{N_{a}}, 0 \frac{N_{b}}{N_{a}}\right)=\left(4 N_{b}, 0\right)=\mathcal{O}_{\mathscr{B}_{N_{b}}} \Rightarrow \chi\left(\mathcal{O}_{\mathscr{S}_{N_{a}}}\right)=\mathcal{O}_{\mathscr{B}_{N_{b}}}\left(i_{1}\right) .
$$

Set $P^{\prime}$ the inverse of $P$ in $\mathscr{B}_{N_{a}}(x, y)$; by definition, $P^{\prime}=\left(x_{p},-y_{p}\right)$.
Then, $\quad \chi\left(P^{\prime}\right)=\chi\left(\left(x_{p},-y_{p}\right)\right)=\left(x_{p}\left(N_{b} / N_{a}\right),-y_{p}\left(N_{b} /\right.\right.$ $\left.\left.N_{a}\right)\right)=\chi(P)^{\prime} \Rightarrow \chi\left(P^{\prime}\right)=\chi(P)^{\prime}\left(i_{2}\right)$.

$$
\begin{aligned}
& \chi(P+Q)=\chi\left(\begin{array}{c}
\frac{1}{2 N_{a}}\left[\left(x_{p}-2 N_{a}\right)\left(x_{q}-2 N_{a}\right)+y_{p} y_{q}\right]+2 N_{a}, \\
\frac{1}{2 N_{a}}\left[y_{p}\left(x_{q}-2 N_{a}\right)+y_{q}\left(x_{p}-2 N_{a}\right)\right]
\end{array}\right), \\
& =\left(\frac{N_{b}}{2 N_{a}^{2}}\left[\left(x_{p}-2 N_{a}\right)\left(x_{q}-2 N_{a}\right)+y_{p} y_{q}\right]+2 N_{b}, \frac{N_{b}}{2 N_{a}^{2}}\left[y_{p}\left(x_{q}-2 N_{a}\right)+y_{q}\left(x_{p}-2 N_{a}\right)\right]\right) . \\
& \chi(P)+\chi(Q)=\left(x_{p} \frac{N_{b}}{N_{a}}, y_{p} \frac{N_{b}}{N_{a}}\right)+\left(x_{q} \frac{N_{b}}{N_{a}}, y_{q} \frac{N_{b}}{N_{a}}\right) \\
& =\left(\begin{array}{c}
\frac{1}{2 N_{b}}\left[\left(x_{p} \frac{N_{b}}{N_{a}}-2 N_{b}\right)\left(x_{q} \frac{N_{b}}{N_{a}}-2 N_{b}\right)+\frac{N_{b}^{2}}{N_{a}^{2}} y_{p} y_{q}\right]+2 N_{b}, \\
\frac{1}{2 N_{b}}\left[y_{p} \frac{N_{b}}{N_{a}}\left(x_{q} \frac{N_{b}}{N_{a}}-2 N_{b}\right)+y_{q} \frac{N_{b}}{N_{a}}\left(x_{p} \frac{N_{b}}{N_{a}}-2 N_{a}\right)\right]
\end{array}\right) \\
& =\left(\begin{array}{c}
\frac{1}{2 N_{b}}\left[\frac{N_{b}}{N_{a}}\left(x_{p}-2 N_{a}\right) \frac{N_{b}}{N_{a}}\left(x_{q}-2 N_{a}\right)+\frac{N_{b}^{2}}{N_{a}^{2}} y_{p} y_{q}\right]+2 N_{b}, \\
\frac{1}{2 N_{b}}\left[y_{p} \frac{N_{b}^{2}}{N_{a}^{2}}\left(x_{q}-2 N_{a}\right)+y_{q} \frac{N_{b}^{2}}{N_{a}^{2}}\left(x_{p}-2 N_{a}\right)\right]
\end{array}\right) \\
& =\left(\begin{array}{c}
\frac{N_{b}}{N_{a}^{2}}\left[\left(x_{p}-2 N_{a}\right)\left(x_{q}-2 N_{a}\right)+y_{p} y_{q}\right]+2 N_{b}, \\
\frac{N_{b}}{N_{a}^{2}}\left[y_{p}\left(x_{q}-2 N_{a}\right)+y_{q}\left(x_{p}-2 N_{a}\right)\right]
\end{array}\right) .
\end{aligned}
$$

$\chi(P+Q)=\chi(P)+\chi(Q)\left(i_{3}\right)$, (it is the relation $\left.\left(i_{3}\right)\right)$. Then, relations $\left(i_{1}\right),\left(i_{2}\right)$, and $\left(\left(i_{3}\right)\right)$ imply that $\mathrm{X}$ is a group morphism Furthermore,

$\operatorname{Ker}(\chi)=\left\{(x, y) \in \mathscr{B}_{N_{a}}(x, y) / \chi(x, y)=\mathcal{O}_{\mathscr{B}_{N_{b}}}\right\}=\left\{\mathcal{O}_{\mathscr{B}_{N_{a}}}\right\}$. $\chi$ is then injective and $\operatorname{Im}(\chi)=\left\{\left(x^{\prime}, y^{\prime}\right) \in \mathscr{B}_{N_{b}}(x, y) /\right.$ $\left.\left(x^{\prime}, y^{\prime}\right)=\chi(x, y) \forall(x, y) \in \mathscr{B}_{N_{a}}(x, y)\right\}=\mathscr{B}_{N_{b}}(x, y)$ since $\forall\left(x^{\prime}, y^{\prime}\right) \in \mathscr{B}_{N_{b}}(x, y), \exists(x, y) \in \mathscr{B}_{N_{a}}(x, y)$ such that $\left(x^{\prime}, y^{\prime}\right)=\chi(x, y) \cdot \chi$ is also surjective. $\Rightarrow \chi$ is bijective.

Since $\chi$ is a homomorphism of group and bijective, then $\chi$ defines an isomorphism of groups. Thus, $\mathscr{B}_{N_{a}}(x, y)$ and $\mathscr{B}_{N_{b}}(x, y)$ are isomorphic.

Definition 1. Given an integer $N$, we $\operatorname{define} \operatorname{Div}(N)$ as the set of all divisors of $N$. That is to say, $\operatorname{Div}(N)=\{x \in \mathbb{Z} / x \mid N\}$.
Example 1. $N=18: \quad \operatorname{Div}(N)=\{1,2,3,6,9,18\} ; \quad \forall N$ prime, $\operatorname{Div}(N)=\{1, N\}$.

Proposition 3

(i) If $k_{i}, k_{j} \in \operatorname{Div}(N)$ such that $k_{i} \times k_{j}=N$, then $\left(\left(k_{i}+k_{j}\right)^{2},\left(k_{i}^{2}-k_{j}^{2}\right)\right), \quad\left(k_{i}\left(k_{j}+1\right)^{2}, k_{i}\left(k_{j}^{2}-1\right)\right) \epsilon$ $\mathscr{B}_{N}(x, y)$.

(ii) If $k_{i}, k_{j} \in \operatorname{Div}(N) \backslash\{1, N\}$ with $k_{i}, k_{j}$ primes, then $k_{j} / k_{i} \mid N$ and $\left(\left(k_{j}+2 k_{i}\right) N_{\alpha}+k_{i} N_{\beta},\left(k_{j}^{2}-k_{i}^{2}\right) N_{\gamma}\right)$ $\in \mathscr{B}_{N}(x, y) \quad$ where $\quad N_{\alpha}=N / k_{i}, \quad N_{\beta}=n / k_{j}$, $N_{\gamma}=N / k_{i} k_{j}$.

(iii) More generally, if $k \mid N \Leftrightarrow((k+2) N+(N / k)$, $\left.\left(k^{2}-1 / k\right) N\right) \in \mathscr{B}_{N}(x, y)$. 
Proof

(i) If $k_{i}, k_{j} \in \operatorname{Div}(N), k_{i} \times k_{j}=N$. $\left(\left(k_{i}+k_{j}\right)^{2},\left(k_{i}^{2}-\right.\right.$ $\left.\left.k_{j}^{2}\right)\right) \in \mathscr{B}_{N}(x, y)\left(\left(k_{i}^{2}-k_{j}^{2}\right)\right)^{2}=\left(\left(k_{i}+k_{j}\right)^{2}\right)^{2}-4 N$ $\left(\left(k_{i}+k_{j}\right)^{2}\right)=\left(k_{i}+k_{j}\right)^{2}\left(k_{j}^{2}+k_{j}^{2}+2 N-4 N\right)=\left(k_{i}+\right.$ $\left.k_{j}\right)^{2}\left(k_{i}-k_{j}\right)^{2}=\left(k_{i}^{2}-k_{j}^{2}\right)^{2}$. Also, $\left(k_{i}\left(k_{j}+1\right)^{2}, k_{i}\left(k_{j}^{2}\right.\right.$ $-1)) \in \mathscr{B}_{N}(x, y) \Leftrightarrow\left(k_{i}\left(k_{j}^{2}-1\right)\right)^{2}=\left(k_{i}\left(k_{j}+1\right)^{2}\right)^{2}-$ $4 N\left(k_{i}\left(k_{j}+1\right)^{2}\right)=k_{i}^{2}\left(k_{j}+1\right)^{2}\left[\left(k_{j}+1\right)^{2}-4 \quad(N /\right.$ $\left.\left.k_{i}\right)\right]=k_{i}^{2}\left(k_{j}+1\right)^{2}\left[\left(k_{j}+1\right)^{2}-4 k_{j}\right]=k_{i}^{2}\left(k_{j}+1\right)^{2}\left[k_{j}^{2}\right.$ $\left.+2 k_{j}-4 k_{j}+1\right]=k_{i}^{2}\left(k_{j}+1\right)^{2}\left(k_{j}-1\right)^{2}=k_{i}^{2}\left(k_{j}^{2}-\right.$ $1)^{2}=\left(k_{i}\left(k_{j}^{2}-1\right)\right)^{2}$.

(ii) If $k_{i}, k_{j} \in \operatorname{Div}(n) \backslash\{1, N\}, k_{j} / k_{i} \mid N \Rightarrow\left(\left(k_{j}+2 k_{i}\right) N_{\alpha}+\right.$ $\left.k_{i} N_{\beta},\left(k_{j}^{2}-k_{i}^{2}\right) N_{\gamma}\right) \in \mathscr{B}_{N}(x, y)$ where $N_{\alpha}=N / k_{i}$, $N_{\beta}=N / k_{j}, N_{\gamma}=N / k_{i} k_{j}$. It comes to verify that $\left(\left(k_{j}+2 k_{i}\right) N_{\alpha}+k_{i} n_{\beta},\left(k_{j}^{2}-k_{i}^{2}\right) N_{\gamma}\right) \quad$ verifies the equation $y^{2}=x^{2}-4 N x$. As $N=k_{j} \times k_{i}, k_{j}, k_{i}$ being prime and $\operatorname{Div}(N)=\left\{1, k_{j}, k_{i}, N\right\}$, then $N_{\alpha}=N / k_{i}=k_{j}, N_{\beta}=N / k_{j}=k_{i}, N_{\gamma}=N / k_{j} k_{i}=1$. We have $\left(\left(k_{j}+2 k_{i}\right) N_{\alpha}+k_{i} N_{\beta},\left(k_{j}^{2}-k_{i}^{2}\right) N_{\gamma}\right)=$ $\left(\left(k_{j}+2 k_{i}\right) N_{\alpha}+k_{i} N_{\beta}, \quad\left(k_{j}^{2}-k_{i}^{2}\right) N_{\gamma}\right)=\left(\left(k_{j}+\right.\right.$ $\left.\left.2 k_{i}\right) k_{j}+k_{i}^{2},\left(k_{j}^{2}-k_{i}^{2}\right) \times 1\right)=\left(\left(k_{j}+k_{i}\right)^{2},\left(k_{j}^{2}-k_{i}^{2}\right)\right)$ $\in \mathscr{B}_{N}(x, y) \Leftrightarrow\left(k_{j}^{2}-k_{i}^{2}\right)^{2}=\left(\left(k_{j}+k_{i}\right)^{2}\right)^{2}-\quad 4 N$ $\left(\left(k_{j}+k_{i}\right)^{2}\right)=\left(k_{j}+k_{i}\right)^{4}-4 N\left(k_{j}+k_{i}\right)^{2}=\left(k_{j}+\right.$ $\left.k_{i}\right)^{2}\left(\left(k_{j}+k_{i}\right)^{2}-4 N\right)=\left(k_{j}+k_{i}\right)^{2}\left(k_{j}^{2}+k_{i}^{2}+2 N-\right.$ $4 N)=\left(k_{j}+k_{i}\right)^{2}\left(k_{j}^{2}+k_{i}^{2}-2 N\right)=\left(k_{j}+k_{i}\right)^{2}\left(k_{j}-\right.$ $\left.k_{i}\right)^{2}=\left(k_{j}^{2}-k_{i}^{2}\right)^{2}\left(\left(k_{j}+2 k_{i}\right) k_{j}+k_{i}^{2},\left(k_{j}^{2}-k_{i}^{2}\right) \times 1\right)=$ $\left(\left(k_{j}+k_{i}\right)^{2},\left(k_{j}^{2}-k_{i}^{2}\right)\right) \quad \in \mathscr{B}_{N}(x, y) \Leftrightarrow\left(k_{j}^{2}-k_{i}^{2}\right)^{2}=$ $\left(\left(k_{j}+k_{i}\right)^{2}\right)^{2}-4 N \quad\left(\left(k_{j}+k_{i}\right)^{2}\right)=\left(k_{j}+k_{i}\right)^{4}-$ $4 N\left(k_{j}+k_{i}\right)^{2}=\left(k_{j}+k_{i}\right)^{2}\left(\left(k_{j}+k_{i}\right)^{2}-4 N\right)=\left(k_{j}+\right.$ $\left.k_{i}\right)^{2}\left(k_{j}^{2}+k_{i}^{2}+2 N-4 N\right)=\left(k_{j}+k_{i}\right)^{2}\left(k_{j}^{2}+k_{i}^{2}-\right.$ $2 N)=\left(k_{j}+k_{i}\right)^{2}\left(k_{j}-k_{i}\right)^{2}=\left(k_{j}^{2}-k_{i}^{2}\right)^{2}$. Hence, $\left(\left(k_{j}+2 k_{i}\right) N_{\alpha}+k_{i} N_{\beta},\left(k_{j}^{2}-k_{i}^{2}\right) N_{\gamma}\right) \in \mathscr{B}_{N}(x, y)$.

(iii) More generally, if $k \mid N$,

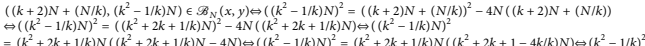

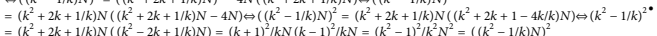

Proposition 4. If $P=\left(x_{p}, y_{p}\right) \in \mathscr{B}_{N}(x, y)_{\left.\right|_{x>4 N}}$, then the following holds:

(i) $\left(x_{p},-y_{p}\right) \in \mathscr{B}_{N}(x, y)$.

(ii) $\left(-x_{p}+4 N, y_{p}\right) \in \mathscr{B}_{N}(x, y)$. (iii) $\left(-x_{p}+4 N,-y_{p}\right) \in \mathscr{B}_{N}(x, y)$.

Proof

(i) $\left(x_{p},-y_{p}\right) \in \mathscr{B}_{N}(x, y)$ is straightforward since it results from the symmetry of $\mathscr{B}_{N}(x, y)$ with respect to the $(O x)$ axis.

(ii) At the point $\left(-x_{p}+4 n, y_{p}\right)$, we have $y_{p}^{2}=$ $\left(-x_{p}+4 N\right)^{2}-4 N\left(-x_{p}+4 N\right) \Leftrightarrow y_{p}^{2}=x_{p}^{2}-8 N x_{p}$ $+16 N^{2}+4 N x_{p}-16 N^{2} \Leftrightarrow \quad y_{p}^{2}=x_{p}^{2}-4 N x_{p}$, $\Rightarrow\left(-x_{p}+4 N, y_{p}\right) \in \mathscr{B}_{N}(x, y)$.

(iii) From the symmetry of $\mathscr{B}_{N}(x, y)$ with respect to the $(O x)$ axis, we obtain $\left(-x_{p}+4 N,-y_{p}\right) \in$ $\mathscr{B}_{N}(x, y)$.

Lemma 1. $\forall a \in \mathbb{Z}$, set $x=(N+a)^{2}$; then, $(x, y) \in \mathscr{B}_{N}(x, y)$ if and only if $a=1$.

Proof. Set $x=(N+a)^{2}$; then, $y^{2}=(N+a)^{4}+4 N$ $(N+a)^{2}=(N+a)^{2}\left[(N+a)^{2}-4 N\right]=(N+a)^{2}\left[N^{2}+2(a\right.$ $\left.-2) N+a^{2}\right]$ which is square if and only if $\delta^{\prime}=(a-2)^{2}-a^{2}=0$, which is impossible except for $a=1$, since $\quad(a-2)^{2}-a^{2}=0 \Leftrightarrow a^{2}-4 a+4-a^{2}=0 \Leftrightarrow 4 a=$ $4 \Leftrightarrow a=1$. This yields that $\forall a \in \mathbb{Z}, x=(N+a)^{2}$ satisfies $y^{2}=$ $x^{2}-4 N x$ if and only if $a=1$.

Remark 1 . Over $\mathbb{Z}, x^{2}-4 N x$ is square only if either $x$ and $x-4 N$ are squares or $x-4 N \mid x$ such that $x /(x-4 N)=k^{2}$, $k \in \mathbb{Z}$.

Theorem 1. Consider $\mathscr{B}_{N}$ over $\mathbb{Z}_{\geq 4 N} \times \mathbb{Z}_{\geq 0}$ denoted as $\mathscr{B}_{N}(x, y)_{\left.\right|_{x \geq 4 N}}$. Then, $\forall P=\left(x_{p}, y_{p}\right) \in \mathscr{B}_{N}(x, y)_{\left.\right|_{x \geq 4 N}}, \quad\left(x_{p}\right.$, $\left.y_{p}\right) \in[4 N, N(N+2)+1] \times\left[0, N^{2}-1\right]$.

Proof. It is not difficult to see that $y^{2}=$ $\left.\left.x^{2}-4 N x \geq 0 \Leftrightarrow x \in\right]-\infty, 0\right] \cup[4 N, \infty$ [. From the Remark 1 , a given $x$ satisfies the condition only if either both $x$ and $x-4 N$ are squares or $(x-4 N) \mid x$ with $x /(x-4 N)=k^{2}$, $k \in \mathbb{Z}$. Considering the fact that any integer $i$ can be written as a function of $N$, that is to say $i=N+a$, where $a=i-N$, if $x$ is square, then $\exists a \in \mathbb{Z}$ such that $x=(N+a)^{2}$. From Lemma 1 , this holds if and only if $a=1$. Now assume there exists $x>(N+1)^{2}$; in this case, $\exists b \in \mathbb{Z}_{\geq 0}$ such that $x=(N+1)^{2}+b$. Then,

$$
\begin{aligned}
x(x-4 N)= & {\left[(N+1)^{2}+b\right]\left[(N+1)^{2}+b-4 N\right]=(N+1)^{4}+2(b-2 N)(N+1)^{2} } \\
& +b(b-4 N) \\
= & b^{2}+\left[2(N+1)^{2}-4 N\right] b+(N+1)^{4}-4 N(N+1)^{2},
\end{aligned}
$$

and (19) is a square if and only if $\delta^{\prime}=(b-2 N)^{2}-b(b-$ $4 N)=0 \Leftrightarrow b^{2}-4 N b+4 N^{2}-b^{2}+4 N b=0 \Leftrightarrow$ $4 N^{2}=0 \Leftrightarrow N=0$, which is absurd since $N \neq 0$.
Also, (19) is a square if and only if $\delta^{\prime}=$ $\left[(N+1)^{2}-2 N\right]^{2}-(N+1)^{4}+4 N(N+1)^{2}=0 \Leftrightarrow(N+1)^{4}$ $-4 N(N+1)^{2}+4 N^{2}-(N+1)^{4}+4 N(N+1)^{2}=0 \Leftrightarrow 4 N^{2}$ 
$=0 \Leftrightarrow N=0$, which is once more absurd since $N \neq 0$. $\Rightarrow \nexists b \in \mathbb{Z}_{\geq 0} / x=(N+1)^{2}+b \quad$ satisfying $\quad y^{2}=x^{2}-4 N x$, $\Rightarrow x \in\left[4 N,(N+1)^{2}\right]$.

If $x=4 N, y^{2}=16 N^{2}-4 N(4 N)=0, \Rightarrow y=0$. Also, if $x=(N+1)^{2}, \quad y^{2}=(N+1)^{2}\left[(N+1)^{2}-4 N\right]=(N+1)^{2}$ $\left(N^{2}+2 N+1-4 N\right)=(N+1)^{2}(N-1)^{2}=\left(N^{2}-1\right)^{2}, \Rightarrow y$ $=N^{2}-1$, and thus $y \in\left[4 N,(N+1)^{2}\right]$.
Hence, $\forall P=\left(x_{p}, y_{p}\right) \in \mathscr{B}_{N}(x, y)_{\left.\right|_{x \geq 4 N}},\left(x_{p}, y_{p}\right) \in[4 N$, $N(N+2)+1] \times\left[0, N^{2}-1\right]$.

\section{Proposition 5}

$\left(p_{1}\right)$ If $N=N_{a} \times N_{b}$, then there exists an injective homomorphism

$$
\begin{aligned}
& \chi_{N_{a} \times N_{b}} \longrightarrow N^{2}: \mathscr{B}_{N_{a}}(x, y) \times \mathscr{B}_{N_{b}}(x, y) \longrightarrow \frac{\mathscr{B}_{N}(x, y) \times \mathscr{B}_{N}(x, y)}{\forall\left(x_{a}, y_{a}\right) \in \mathscr{B}_{N_{a}}(x, y),\left(x_{b}, y_{b}\right) \in \mathscr{B}_{N_{b}}(x, y)}, \\
& \chi_{N_{a} \longrightarrow N}\left(x_{a}, y_{a}\right)=\left(N_{b} x_{a}, N_{b} y_{a}\right) \in \mathscr{B}_{N}, \\
& \chi_{N_{b} \longrightarrow N}\left(x_{b}, y_{b}\right)=\left(N_{a} x_{b}, N_{a} y_{b}\right) \in \mathscr{B}_{N} .
\end{aligned}
$$

$\left(p_{2}\right)$ More generally, there is an injective homomorphism

$$
\begin{aligned}
& \chi_{\prod_{i=1}^{z} N \longrightarrow N^{z}}: \mathscr{B}_{N_{a}}(x, y) \times \mathscr{B}_{N_{b}}(x, y) \times \cdots \times \mathscr{B}_{N_{z}}(x, y) \longrightarrow \frac{\prod_{i=1}^{z} \mathscr{B}_{N}(x, y)}{\forall\left(x_{N_{i}}, y_{N_{i}}\right) \in \mathscr{B}_{N_{i}}(x, y)}, \\
& \chi \prod_{i=1}^{z} N_{i} \longrightarrow n^{z}\left(x_{N_{i}}, y_{N_{i}}\right)=\prod_{i=1, \widehat{j}}^{z} N_{i}\left(x_{N_{i}}, y_{N_{i}}\right) .
\end{aligned}
$$

Proof

$\left(p_{1}\right)$ Set $N=N_{a} \times N_{b}$ and consider $\mathscr{B}_{N_{a}}(x, y)$, $\mathscr{B}_{N_{b}}(x, y)$ and $\mathscr{B}_{N}(x, y)$ and consider the following morphism:

$$
\begin{aligned}
& \chi_{N_{a} \times N_{b}} \longrightarrow N^{2}: \mathscr{B}_{N_{a}}(x, y) \times \mathscr{B}_{N_{b}}(x, y) \\
& \quad \longrightarrow \mathscr{B}_{N}(x, y) \times \mathscr{B}_{N}(x, y) \\
& \quad\left(\left(x_{a}, y_{a}\right),\left(x_{b}, y_{b}\right)\right) \mapsto\left(\left(x_{a} \frac{N}{n_{a}}, y_{a} \frac{N}{N_{a}}\right),\left(x_{b} \frac{N}{N_{b}}, y_{b} \frac{N}{N_{b}}\right)\right) .
\end{aligned}
$$

We clearly see that it is a morphism since it splits into morphisms $\chi_{N_{a} \rightarrow N}$ and $\chi_{N_{a}} \longrightarrow N$ that are already known to be group morphisms from Proposition 2. It stays now to prove the injection. Given $\left(P_{1}, P_{2}\right) \in$ $\mathscr{B}_{N_{a}}(x, y) \times \mathscr{B}_{N_{b}}(x, y), \quad\left(P_{1}^{\prime}, P_{2}^{\prime}\right) \in \mathscr{B}_{N_{a}}(x, y) \times$ $\mathscr{B}_{N_{b}}(x, y) \quad$ such that $\chi_{N_{a} \times N_{b}} \longrightarrow N^{2}\left(P_{1}, P_{2}\right)=$ $\chi_{N_{a} \times N_{b}} \longrightarrow N^{2}\left(P_{1}^{\prime}, P_{2}^{\prime}\right)$ where $P_{1} \stackrel{\chi_{a} \times N_{b}}{=}\left(x_{a}, y_{a}\right), P_{2}=\left(x_{b}\right.$, $\left.y_{b}\right), P_{1}^{4}=\left(x_{a}^{\prime}, y_{a}^{\prime}\right)$ and $P_{2}^{\prime}=\left(x_{b}^{\prime}, y_{b}^{\prime}\right)$. Then,

$$
\begin{aligned}
& \left(\left(x_{a} \frac{N}{N_{a}}, y_{a} \frac{N}{N_{a}}\right),\left(x_{b} \frac{N}{N_{b}}, y_{b} \frac{N}{N_{b}}\right)\right)=\left(\left(x_{a}^{\prime} \frac{N}{N_{a}}, y_{a}^{\prime} \frac{N}{N_{a}}\right),\left(x_{b}^{\prime} \frac{N}{N_{b}}, y_{b}^{\prime} \frac{N}{N_{b}}\right)\right) \\
& \Rightarrow\left\{\begin{array} { l } 
{ ( x _ { a } \frac { N } { N _ { a } } , y _ { a } \frac { N } { N _ { a } } ) = ( x _ { a } ^ { \prime } \frac { N } { N _ { a } } , y _ { a } ^ { \prime } \frac { N } { N _ { a } } ) } \\
{ ( x _ { b } \frac { N } { N _ { b } } , y _ { b } \frac { N } { N _ { b } } ) = ( x _ { b } ^ { \prime } \frac { N } { N _ { b } } , y _ { b } ^ { \prime } \frac { N } { N _ { b } } ) }
\end{array} \Leftrightarrow \left\{\begin{array}{l}
x_{a} \frac{N}{N_{a}}=x_{a}^{\prime} \frac{N}{N_{a}} \\
x_{b} \frac{N}{N_{b}}=x_{b}^{\prime} \frac{N}{N_{b}}
\end{array},\left\{\begin{array}{l}
y_{a} \frac{N}{N_{a}}=y_{a}^{\prime} \frac{N}{N_{a}} \\
y_{b} \frac{N}{N_{b}}=y_{b}^{\prime} \frac{N}{n_{b}}
\end{array}\right.\right.\right. \\
& \Rightarrow \begin{cases}x_{a}=x_{a}^{\prime}, & y_{a}=y_{a}^{\prime}, \\
x_{b}=x_{b}^{\prime}, & y_{b}=y_{b}^{\prime} .\end{cases}
\end{aligned}
$$


Hence, $\chi_{N_{a} \times N_{b}} \longrightarrow N^{2}\left(P_{1}, P_{2}\right)=\chi_{N_{a} \times N_{b}} \longrightarrow N^{2}\left(P_{1}^{\prime}, P_{2}^{\prime}\right) \Leftrightarrow$ $P_{1}=P_{1}^{\prime}$ and $P_{2}=P_{2}^{\prime}$, hence the injection.

$\left(p_{2}\right)$ This comes straightforward from $\left(p_{1}\right)$.
Example 2. We consider the two hyperbolas plotted in Figure 1.

(i) $N=221=N_{a} \times N_{b}=13 \times 17$ :

$$
\begin{aligned}
\mathscr{B}_{221}(x, y)_{\mid x \geq 4 N}= & \{(884,0),(900,120),(3332,2856),(4212,3744),(49284,48840)\}, \\
\mathscr{B}_{13}(x, y)_{\mid x \geq 4 N}= & \{(52,0),(196,168)\}, \\
\mathscr{B}_{17}(x, y)= & \{(68,0),(324,288)\}, \\
\chi_{13 \times 17 \longrightarrow 221^{2}}: & \mathscr{B}_{13}(x, y)_{\mid x \geq 4 N} \times \mathscr{B}_{17}(x, y)_{\mid x \geq 4 N} \longrightarrow \mathscr{B}_{221}(x, y)_{\mid x \geq 4 N} \times \mathscr{B}_{221}(x, y)_{\mid x \geq 4 N} \\
& \cdot\left(\left(x_{a}, y_{a}\right),\left(x_{b}, y_{b}\right)\right) \mapsto\left(\left(17 x_{1}, 17 y_{1}\right),\left(13 x_{b}, 13 y_{b}\right)\right), \\
\chi_{13 \longrightarrow 221}(52,0)= & (17 \times 52,17 \times 0)=(884,0) \in \mathscr{B}_{221}(x, y), \\
\chi_{13 \longrightarrow 221}(196,168)= & (17 \times 196,17 \times 168)=(3332,2856) \in \mathscr{B}_{221}(x, y), \\
\chi_{17 \longrightarrow 221}(68,0)= & (13 \times 68,13 \times 0)=(884,0) \in \mathscr{B}_{221}(x, y), \\
\chi_{17 \longrightarrow 221}(324,288)= & (13 \times 324,13 \times 288)=(4212,3744) \in \mathscr{B}_{221}(x, y) .
\end{aligned}
$$

(ii) $N=210=2 \times 3 \times 5 \times 7$ :

$$
\begin{aligned}
& \text { (840, 0), (841, 29), (845, 65), (847, 77), (864, 144), } \\
& \text { (867, 153), (875, 175), (896, 224), (945, 315), (961, 341), (968, 352) }(1000,400) \text {, } \\
& (1029,441),(1083,513),(1120,560),(1183,637),(1215,675) \text {, } \\
& \text { (1352, 832), (1369, 851), (1445, 935), (1512, 1008), (1681, 1189), (1715, 1225), } \\
& \text { (1920, 1440), (2023, 1547), (2209, 1739), (2541, 2079), (2645, 2185), } \\
& \text { (2888, 2432), (3375, 2925), (3584, 3136), (4107, 3663), (4840, 4400), (5329, 4891), } \\
& (6727,6293),(7776,7344),(9245,8815),(11449,11021),(15123,14697) \\
& \text {, (22472, 22048), (44521, 44099) } \\
& \mathscr{B}_{2}(x, y)_{\mid x \geq 4 N}=\{(8,0),(9,3)\} \text {, } \\
& \mathscr{B}_{3}(x, y)=\{(12,0),(16,8)\}, \\
& \mathscr{B}_{5}(x, y)=\{(20,0),(36,24)\} \\
& \mathscr{B}_{7}(x, y)=\{(28,0),(64,48)\}, \\
& \chi_{2 \times 3 \times 5 \times 7 \longrightarrow 210^{4}}: \mathscr{B}_{2}(x, y) \times \mathscr{B}_{3}(x, y) \times \mathscr{B}_{5}(x, y) \times \mathscr{B}_{7}(x, y) \longrightarrow \\
& \prod_{i=1}^{4} \mathscr{B}_{210}(x, y)=\mathscr{B}_{210}(x, y) \times \cdots \times \mathscr{B}_{210}(x, y) \\
& \cdot\left(\left(x_{a}, y_{a}\right),\left(x_{b}, y_{b}\right),\left(x_{c}, y_{c}\right),\left(x_{d}, y_{d}\right)\right) \mapsto\left(\begin{array}{c}
\left(105 x_{1}, 105 y_{1}\right),\left(70 x_{2}, 70 y_{2}\right), \\
\left(42 x_{3}, 42 y_{3}\right),\left(30 x_{4}, 30 y_{4}\right)
\end{array}\right), \\
& \chi_{2 \longrightarrow 210}(8,0)=(105 \times 8,105 \times 0)=(840,0) \in \mathscr{B}_{210}(x, y) \text {, } \\
& \chi_{2 \longrightarrow 210}(9,3)=(105 \times 9,105 \times 3)=(945,315) \in \mathscr{B}_{210}(x, y) \text {, } \\
& \chi_{3 \longrightarrow 210}(12,0)=(70 \times 12,70 \times 0)=(840,0) \in \mathscr{B}_{210}(x, y) \text {, } \\
& \chi_{3 \longrightarrow 210}(16,8)=(70 \times 16,70 \times 8)=(1120,560) \in \mathscr{B}_{210}(x, y) \text {, } \\
& \chi_{5 \longrightarrow 210}(20,0)=(42 \times 20,42 \times 0)=(840,0) \in \mathscr{B}_{210}(x, y) \text {, } \\
& \chi_{5 \longrightarrow 210}(36,24)=(42 \times 36,42 \times 24)=(1512,1008) \in \mathscr{B}_{210}(x, y) \text {, } \\
& \chi_{7 \longrightarrow 210}(28,0)=(30 \times 28,30 \times 0)=(840,0) \in \mathscr{B}_{210}(x, y) \text {, } \\
& \chi_{7 \longrightarrow 210}(64,48)=(30 \times 64,30 \times 48)=(1920,1440) \in \mathscr{B}_{210}(x, y) .
\end{aligned}
$$




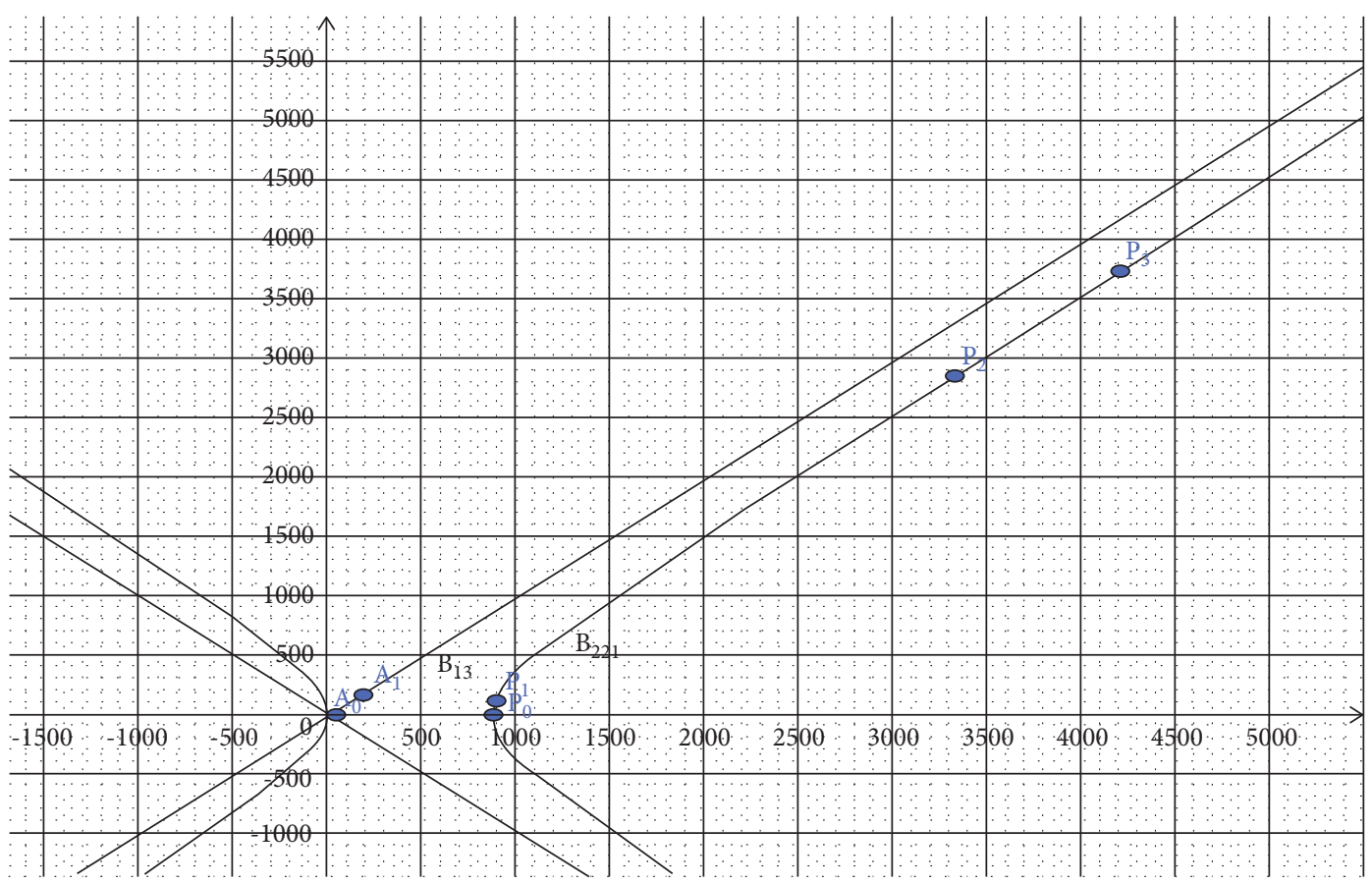

FIGURE 1: $\mathscr{B}_{13}(x, y)_{\left.\right|_{x \geq 52}}$ and $\mathscr{B}_{221}(x, y)_{\left.\right|_{x \geq 884}}$.

Definition 2. A prime divisor of an integer $n$ is any prime number $p \in \operatorname{Div}(n)$. We denote $\pi_{p}(n)$ as the set of all prime divisors of $n$ and $\left|\pi_{p}(n)\right|$ as the number of prime divisors of $n$.

Example 3. 5 is a prime divisor of 40 since $5 \in \operatorname{Div}(40)=\{1,2,4,5,8,10,20,40\} . \quad \pi_{p}(40)=\{2,5\} \quad$ and $\left|\pi_{p}(40)\right|=2$.

Proposition 6. Set $N=\prod_{i=1}^{\delta} p_{i}, p_{i}$ primes and consider $\mathscr{B}_{N}(x, y)_{\mid x \geq 4 N}=\left\{(x, y) \in \mathbb{Z}_{\geq 4 N} \times \mathbb{Z}_{\geq 0} / y^{2}=x^{2}-4 N x\right\}$. Set $n=\left|\pi_{p}(N)\right|$ and $U_{n}$ as the cardinal of $\mathscr{B}_{N}(x, y)_{\mid x \geq 4 N}$. Then, $U_{n}=3 U_{n-1}-1 \forall n \geq 1$ with $U_{0}=1$. In this case, we have the induction relation $U_{n+2}=2 U_{n+1}+3 U_{n}-2$ and the sum of cardinals of $\mathscr{B}_{N_{i}}(x, y)_{x \geq 4 N_{i}}$ given by the general term $U_{n}$ is $S_{n}=\sum_{i=1}^{n} U_{i}=(1 / 2) n-(3 / 4)\left(1-3^{n}\right)$.

Proof. $\quad n=\left|\pi_{p}(N)\right|, \quad U_{n}=\left|\mathscr{B}_{N}(x, y)_{\mid x \geq 4 N}\right|=3 U_{n-1}-1$ where $\quad U_{0}=1$, i.e., $\quad\left|\mathscr{B}_{m}(x, y)_{\mid x \geq 4 m}\right|=U_{\left|\pi_{p}(m)\right|}=$ $3 U_{\left|\pi_{p}(m)\right|-1}-1$.

By induction on $n$, we have

For $n=1, U_{1}=3 U_{0}-1=2$ (true).
For $n=2, U_{2}=3 U_{1}-1=5$ (true).

Assume the relation to be true for $n$, i.e., $U_{n}=3 U_{n-1}-1$, and let us show the relation to be true for $n+1$.

$$
\begin{aligned}
U_{\left|\pi_{p}(m)\right|+1} & =3\left(3 U_{\left|\pi_{p}(m)\right|-1}-1\right)-1 \\
& =3 U_{\left|\pi_{p}(m)\right|}-1=3 U_{n}-1=U_{n+1} .
\end{aligned}
$$

By the same,

$$
\begin{aligned}
& U_{n+1}=3\left(U_{n}-1\right)+2=3 U_{n}-1, \\
& U_{n+2}=3\left(U_{n+1}-1\right)+2=3 U_{n+1}-1 .
\end{aligned}
$$

By substituting (27) and (28), we obtain

$$
\begin{gathered}
U_{n+2}+U_{n+1}=3 U_{n+1}-1+3 U_{n}-1=3 U_{n+1}+3 U_{n}-2 \\
\Rightarrow U_{n+2}=2 U_{n+1}+3 U_{n}-2 .
\end{gathered}
$$

For $S_{n}$ :

$$
\begin{aligned}
& U_{1}=3 U_{0}-1, \\
& U_{2}=3 U_{1}-1=3\left(3 U_{0}-1\right)-1=3^{2} U_{0}-3-1, \\
& U_{3}=3 U_{2}-1=3\left(3^{2} U_{0}-3-1\right)-1=3^{3} U_{0}-3^{2}-3-1, \\
& U_{4}=3 U_{3}-1=3\left(3^{3} U_{0}-3^{2}-3-1\right)-1=3^{4} U_{0}-3^{3}-3^{2}-3-1,
\end{aligned}
$$




$$
\begin{aligned}
U_{5}= & 3 U_{4}-1=3\left(3^{4} U_{0}-3^{3}-3^{2}-3-1\right)-1=3^{5} U_{0}-3^{4}-3^{3}-3^{2}-3-1, \\
U_{6} & =3 U_{5}-1=3\left(3^{5} U_{0}-3^{4}-3^{3}-3^{2}-3-1\right)-1=3^{6} U_{0}-3^{5}-3^{4}-3^{3}-3^{2}-3-1, \\
& \vdots \\
U_{n} & =3 U_{n-1}-1=3^{n} U_{0}-\sum_{i=0}^{n-1} 3^{i}=3^{n}-\sum_{i=0}^{n-1} 3^{i} \text { since } U_{0}=1 \\
\sum_{j=1}^{n} U_{j}=\sum_{j=1}^{n}\left(3 U_{j-1}-1\right) & =\sum_{j=1}^{n}\left(3^{j}-\sum_{i=0}^{j-1} 3^{i}\right) \\
& =\sum_{j=1}^{n} 3^{j}-\sum_{j=1}^{n} \sum_{i=0}^{j-1} 3^{i}=\sum_{j=1}^{n} 3^{j}-\sum_{j=1}^{n} \frac{1-3^{j}}{1-3}=\sum_{j=1}^{n} 3^{j}+\frac{1}{2} \sum_{j=1}^{n}\left(1-3^{j}\right)=\sum_{j=1}^{n} 3^{j}+\frac{1}{2} n-\frac{1}{2} \sum_{j=1}^{n} 3^{j} \\
& =\frac{1}{2} n+\frac{1}{2} \sum_{j=1}^{n} 3^{j}=\frac{1}{2}\left[n-\frac{3}{2}\left(1-3^{n}\right)\right] \Rightarrow S_{n}=\sum_{j=1}^{n} U_{j}=\frac{1}{2} n-\frac{3}{4}\left(1-3^{n}\right) .
\end{aligned}
$$

Example 4

\section{Verification:}

(i) $m=253, \quad n=\left|\pi_{p}(m)\right|=2$, and $U_{2}=3 U_{1}-1$,

knowing $U_{1}=3 U_{0}-1=3(1)-1=2$ since $U_{0}=1$.

Thus, $U_{2}=3(2)-1=5 \Rightarrow\left|\mathscr{B}_{253}(x, y)_{\mid x \geq 4 m}\right|=5$.

$$
\begin{aligned}
& \mathscr{B}_{253}(x, y)_{\mid x \geq 4 m}=\{(1012,0),(1156,408),(3312,2760),(6336,5808),(64 \quad 516,64 \quad 008)\}, \\
& \left|\mathscr{B}_{253}(x, y)_{\mid x \geq 4 m}\right|=5 \text {. }
\end{aligned}
$$

(ii) $m=30$ and $n=\left|\pi_{p}(m)\right|=3, U_{3}=3 U_{2}-1$ knowing

\section{Verification:}

$U_{2}=5$, and thus $U_{3}=3(5)-1=14 \Rightarrow$

$\left|\mathscr{B}_{30}(x, y)_{\mid x \geq 4 m}\right|=14$.

$$
\mathscr{B}_{30}(x, y)_{\mid x \geq 4 m}=\left\{\begin{array}{c}
(120,0),(121,11),(125,25),(128,32), \\
(135,45),(147,63),(160,80),(169,91), \\
(216,144),(245,175),(289,221),(363,297),(512,448),(961,899)
\end{array}\right\},
$$

and thus $\left|\mathscr{B}_{30}(x, y)_{\mid x \geq 4 m}\right|=14$.

(iii) $m=2002, \quad n=\left|\pi_{p}(m)\right|=4$, and $U_{4}=3 U_{3}-1$ knowing $U_{3}=14$, and thus $U_{4}=3(14)-1=$ $41 \Rightarrow\left|\mathscr{B}_{2002}(x, y)_{\mid x \geq 4 m}\right|=41$.

Verification: 


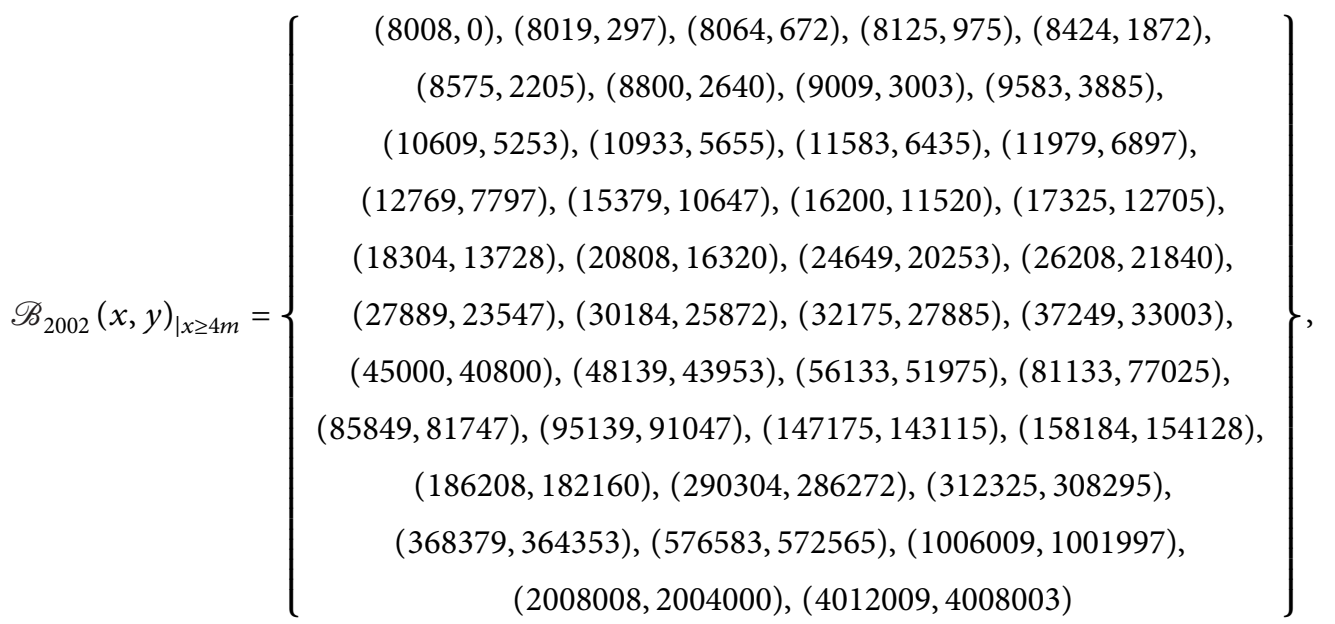

and thus $\left|\mathscr{B}_{2002}(x, y)_{\mid x \geq 4 m}\right|=41$.

\section{Theorem 2}

(1) $N$ is prime if and only if $\left|\mathscr{B}_{N}(x, y)_{\mid x \geq 4 N}\right|=2$.

(2) If $N=N_{a} \times N_{b}$ where $N_{a}$ and $N_{b}$ are distinct primes, then $\operatorname{Card}\left(\mathscr{B}_{N}(x, y)_{\mid x \geq 4 N}\right)=5$.

(3) If $N=N_{a} \times N_{b} \times N_{c}$ where $N_{a}, N_{b}$ and $N_{c}$ are distinct primes, then $\operatorname{Card}\left(\mathscr{B}_{N}(x, y)_{\mid x \geq 4 N}\right)=14$.

(4) If $N=N_{a} \times N_{b} \times N_{c} \times N_{d}$ where $N_{a}, N_{b}, N_{c}$, and $N_{d}$ are distinct primes, then $\operatorname{Card}\left(\mathscr{B}_{N}\right.$ $\left.(x, y)_{\mid x \geq 4 N}\right)=41$.

(5) $\operatorname{Card}\left(\mathscr{B}_{N}(x, y)_{\mid x \in \mathbb{Z}}\right)=4\left(\operatorname{Card}\left(\mathscr{B}_{N}(x, y)_{\mid x \geq 4 N}\right)-\right.$ $1)+1_{(4 N, 0)}+1_{(0,0)}=4\left(\operatorname{Card}\left(\mathscr{B}_{N}(x, y)_{\mid x \geq 4 N}\right)\right)-2$.

(6) Set $N_{a}$ as a prime number, and $\alpha \in \mathbb{Z}_{\geq 0}$ such that $N=N_{a}^{\alpha}$. Then, $\operatorname{Card}\left(\mathscr{B}_{N}(x, y)_{\mid x \geq 4 N}\right)=\alpha+1$, and $\operatorname{Card}\left(\mathscr{B}_{N}(x, y)_{\mid x \in \mathbb{Z}}\right)=4 \alpha+2$.

(7) If $N \neq N_{a}^{\alpha},(p, \alpha) \in \mathbb{Z}_{\geq 0}^{2}, \quad \operatorname{Card}\left(\mathscr{B}_{N}(x, y)_{\mid x \geq 4 N}\right)=$ $\operatorname{Card}(\operatorname{Div}(N))+\sum_{i, j \in \operatorname{Div}(N) \backslash\{1, N\} / i \times j=N}\left(1-\delta_{i j}\right)$.

More generally, $\quad \operatorname{Card}\left(\mathscr{B}_{N}(x, y)_{\mid x \in \mathbb{Z}}\right)=$ $4\left(\operatorname{Card}(\operatorname{Div}(N))+\sum_{i, j \in \operatorname{Div}(N) \backslash\{1, N\} / i \times j=N}\left(1-\delta_{i j}\right)\right)-2$.

Proof

(i) (2), (3), (6), and (9) come straightforward from the Proposition 6. Nevertheless, we give other proofs for (2) and (3) using injective homomorphisms.

(1) Assume $N_{a}$ prime; then, $\operatorname{Div}\left(N_{a}\right)=\left\{1, N_{a}\right\}$. The only injective homomorphisms in $\mathscr{B}_{N_{a}}(x, y)_{\mid x \geq 4 N_{a}}$ are $\chi_{1 \longrightarrow N_{a}}$ giving the point $\left(4 N_{a}, 0\right)$ and the trivial automorphism $\quad \chi_{N} \longrightarrow N_{a}$ giving the point $\left(N_{a}\left(N_{a}+2\right)+1, N_{a}^{2^{a}}-1\right)$ from Proposition 3. As $1 \mid N_{a}$ and $N_{a} \mid N_{a} \Rightarrow \mathscr{B}_{N_{a}}(x, y)_{\mid x \geq 4 N_{a}}=\left\{\left(4 N_{a}, 0\right)\right.$, $\left.\left(N_{a}\left(N_{a}+2\right)+1, N_{a}^{2}-1\right)\right\}, \quad\left|\mathscr{B}_{N_{a}}(x, y)_{\mid x \geq 4 N_{a}}\right|=2$. Now assume $\left|\mathscr{B}_{N_{a}}(x, y)_{\mid x \geq 4 N_{a}}\right|=2$, and we know that $\quad\left(4 N_{a}, 0\right)$ and $\left(\left(N_{a}+1\right)^{2}, N_{a}^{2}-1\right) \epsilon$ $\mathscr{B}_{N_{a}}(x, y)_{\mid x \geq 4 N_{a}}$; that is to say, $\mathscr{B}_{N_{a}}(x, y)_{\mid x \geq 4 N_{a}}=$ $\left\{\left(4 N_{a}, 0\right),\left(\left(N_{a}^{a}+1\right)^{2}, N_{a}^{2}-1\right)\right\}$. From Proposition $6, U_{n}=3 U_{n-1}-1 \forall n \geq 1$ with $U_{0}=1$, and since the cardinal $U_{n}=2, \Rightarrow n=1$, i.e., $U_{n}=3 U_{0}-1=2, N_{a}$ is prime.

(2) If $N=N_{a} \times N_{b}$ where $N_{a}$ and $N_{b}$ are distinct primes, then $\operatorname{Div}(N)=\left\{1, N_{a}, N_{b}, N\right\}$. We then have the following injective morphisms $\chi_{1 \longrightarrow N}$, $\chi_{N_{a}} \longrightarrow N, \chi_{N_{b}} \longrightarrow N$ and the trivial automorphism $\chi_{N \rightarrow N}$. As $\operatorname{Div}\left(N_{a}\right)=\left\{1, N_{a}\right\}$ and $\operatorname{Div}\left(N_{b}\right)=$ $\left\{1, N_{b}\right\}$, then $\left|\mathscr{B}_{N_{a}}(x, y)_{\mid x \geq 4 N_{a}}\right|=2$ and $\mid \mathscr{B}_{N_{b}}$ $(x, y)_{\mid x \geq 4 N_{b}} \mid=2$; in this case, $\mathscr{B}_{N_{a}}(x, y)_{\mid x \geq 4 N_{a}}=$ $\left\{\left(4 N_{a}, 0\right),\left(N_{a}\left(N_{a}+2\right)+1, N_{a}^{2}-1\right)=P_{N_{a}}\right\}, \mathscr{B}_{N_{b}}(x$, $y)_{\mid x \geq 4 N_{b}}=\left\{\left(4 N_{b}, 0\right),\left(N_{b}\left(N_{b}+2\right)+1, N_{b}^{2}-1\right)=\right.$ $\left.P_{N_{b}}\right\} . \chi_{N \longrightarrow N}(x, y)=\left(N(N+2)+1, N^{2}-1\right)=P_{N}$, $\chi_{1 \longrightarrow N}(x, y)=\chi_{N_{a} \longrightarrow N}\left(4 N_{a}, 0\right)=\chi_{N_{b} \longrightarrow N}\left(4 N_{b}, 0\right)=$ $(4 N, 0)=P_{0}, \chi_{N_{a} \longrightarrow N}\left(P_{N_{a}}\right)=P_{1}, \chi_{N_{b}} \longrightarrow N \quad\left(P_{N_{b}}\right)=$ $P_{2}$, and from the Proposition 3, $P_{N_{a}+N_{b}}=$ $\left(\left(N_{a}+N_{b}\right)^{2}, N_{a}^{2}-N_{b}^{2}\right)=P_{3} \Rightarrow \mathscr{B}_{N}(x, y)_{\mid x \geq 4 N}=\left\{P_{0}\right.$, $\left.P_{1}, P_{2}, P_{3}, P_{N}\right\}$. Hence, Card $\left(\mathscr{B}_{N}(x, y)_{\mid x \geq 4 N}\right)=5$.

(5) From Proposition $4, \forall P=\left(x_{p}, y_{p}\right) \in \mathbb{Z}_{\geq 4 N^{2}} \times \mathbb{Z}_{\geq 0}$, $P \in \mathscr{B}_{N}(x, y)$; then, $\left(\left(x_{p},-y_{p}\right),\left(-x_{p}+4 N, y_{p}\right)\right.$, $\left.\left(-x_{p}+4 N,-y_{p}\right)\right) \in \mathscr{B}_{N}^{3}(x, y)$; as $\forall x_{p} \geq 4 N,-x_{p}+$ $4 N \leq 0$ except for the point $(4 N, 0)$ which gives the point itself and the point $(0,0)$, then each point on $\mathscr{B}_{N}(x, y)_{\mid x \geq 4 N} \backslash\{(4 N, 0)\}$ leads to 4 points on $\mathscr{B}_{N}(x, y)_{\mid \mathbb{Z}} \Rightarrow \operatorname{Card}\left(\mathscr{B}_{N}(x, y)_{\mid x \in \mathbb{Z}}\right)=4\left(\operatorname{Card}\left(\mathscr{B}_{N}\right.\right.$ $\left.\left.(x, y)_{\mid x \geq 4 N}\right)-1_{(4 N, 0)}\right)+1_{(4 N, 0)}+1_{(0,0)}$, i.e., $\operatorname{Card}\left(\mathscr{B}_{N}\right.$ $\left.(x, y)_{\mid x \in \mathbb{Z}}\right)=4\left(\operatorname{Card}\left(\mathscr{B}_{N}(x, y)_{\mid x \geq 4 N}\right)\right)-2$.

(6) By induction: if $\alpha=1, N=N_{a}$, then from (2), $\left|\mathscr{B}_{N_{a}}(x, y)_{\mid x \geq 4 N_{a}}\right|=2=\alpha+1 \quad$ (true). If $\alpha=2$, $N=N_{a}^{2}$, from $\quad$ Proposition 3, since $\operatorname{Div}(N)=\operatorname{Div}\left(N_{a}^{\alpha}\right)=\left\{1, N_{a}, N\right\}$, we have the following injective morphisms $\chi_{1 \longrightarrow N}, \chi_{N_{a} \longrightarrow N}$ and the trivial automorphism $\chi_{N \rightarrow N}$ yielding to respectively 3 points $(4 N, 0),\left(N_{a}\left(N_{a}+1\right)^{2}, N_{a}\left(N_{a}^{2}-1\right)\right)$, and $\left((N+1)^{2}, N^{2}-1\right)$. Thus, $\left|\mathscr{B}_{N}(x, y)_{\mid x \geq 4 N}\right|=3=\alpha+1$ (true). Now assume the assumption to be true for $\alpha$, i.e., for $N=N_{a}^{\alpha}$ with $\operatorname{Div}(N)=\left\{1, N_{a}, \ldots, N_{a}^{\alpha-1}, N\right\}$, $\left|\mathscr{B}_{N}(x, y)_{\mid x \geq 4 N}\right|=\alpha+1$. Let us prove that to be also true for $\alpha+1$. If $N=N_{a}^{\alpha} \Rightarrow N \cdot N_{a}=N_{a}^{\alpha+1}$. In this case, $\operatorname{Div}(N)=\left\{1, N_{a}, \ldots, N_{a}^{\alpha-1}, N_{a}^{\alpha}, N\right\}$. To the injective 
morphisms obtained with $\alpha$ from the induction hypothesis, there is now the new injective homomorphism got with the multiplication of $N$ by $N_{a}$. In this case, $\left|\mathscr{B}_{N}(x, y)_{\mid x \geq 4 N}\right|=\alpha+1+1=\alpha+2$. One deduces from $(11)$ that $\operatorname{Card}\left(\mathscr{B}_{N}(x, y)_{\mid x \in \mathbb{Z}}\right)=$ $4\left(\operatorname{Card}\left(\mathscr{B}_{N}(x, y)_{\mid x \geq 4 N}\right)\right)-2=4(\alpha+1)-2=4 \alpha+2$.

(7) From Proposition 5, $\forall i \in \operatorname{Div}(N), \exists x_{i \rightarrow N}: \mathscr{B}_{i}(x$, $y) \longrightarrow \mathscr{B}_{N}(x, y)$, it is clear that $\operatorname{Card}(\operatorname{Div}(N)) \leq$ $\operatorname{Card}\left(\mathscr{B}_{N}(x, y)_{\mid x \geq 4 N}\right)$. By the same, we have injective homomorphisms obtained by composition $\forall i, j \in \operatorname{Div}(N) \backslash\{1, N\}, i \times j=N \Rightarrow\left((i+j)^{2}, i^{2}-j^{2}\right) \epsilon$ $\mathscr{B}_{N}(x, y)$ and we have the injective homomorphisms $\chi_{i \times j \rightarrow N}$ for each case. Therefore, Card $\left(\mathscr{B}_{N}\right.$ $\left.(x, y)_{\mid x \geq 4 N}\right)=\operatorname{Card}(\operatorname{Div}(N))+\operatorname{Card}(\{(i, j) \epsilon$ $\left.\left.\operatorname{Div}(N)^{2} / i \cdot j=N\right\}\right)$.

Then, for each $(i, j)$ verifying this condition, we can express this condition according to the Kronecker symbol. Indeed for the injective homomorphism $\chi_{i \cdot j \longrightarrow N}$, if $i=j$, there is no point since $i \cdot j=N$. If $i \neq j$, we have a point since $i \cdot j \mid N$. Hence, $\left.\operatorname{Card}\left(\mathscr{B}_{N}(x, y)_{\mid x \geq 4 N}\right)=\operatorname{Card}(\operatorname{Div}(N))\right)+$ $\sum_{i, j \in \operatorname{Div}(N) \backslash\{1, N\} / i \times j=N}\left(1-\delta_{i j}\right)$. From (11), we deduce that $\operatorname{Card}\left(\mathscr{B}_{N}(x, y)_{\mid x \in \mathbb{Z}}\right)=4\left(\operatorname{Card}(\operatorname{Div}(N))+\sum_{i, j \in \operatorname{Div}(N) \backslash}\right.$ $\left.\{1, N\} / i \times j=N\left(1-\delta_{i j}\right)\right)-2$.

Proposition 7. The cardinal of $\mathscr{B}_{N}$ over $\mathbb{Z}$ is given by the sequence $U_{n}^{*}=6\left(2 U_{n-1}-1\right)$, and the sum of this sequence is $S_{n}^{*}=2 n-3\left(1-3^{n}\right)$.

Proof. These results are straightforward from Theorem 2. $U_{n}^{*}=4 U_{n}-2$ from the Proposition 6. Then, $U_{n}^{*}=$ $4\left(3 U_{n-1}-1\right)-2=12 U_{n-1}-6=6\left(2 U_{n-1}-1\right)$. Also, $S_{n}^{*}=$ $4 S_{n}=4\left((1 / 2) n-(3 / 4)\left(1-3^{n}\right)\right)=2 n-3\left(1-3^{n}\right)$.

Plots of $U_{n}, S_{n}, U_{n}^{*}$ and, $S_{n}^{*}$ for different values of nare based on the dataset given by Table 1 for different distinct primes.

Comment on the plots on Figure 2 plotted with data from Table 1, from the first plot on the left corresponding to cardinals and sums of cardinals of $\mathscr{B}_{n}(x, y)_{\mid x \geq 4 n}$, we observe that the number of solutions grow quasi-exponentially with the number of distinct prime factors o $n$. In other words, the more distinct the prime factors of $n$, the bigger the algebraic set of $\mathscr{B}_{n}$. Also, from the second plot (on the right), we observe that asymptotically, $\mathscr{B}_{n}(x, y)_{\mid x \geq 4 n}$ and $\mathscr{B}_{n}(x, y)_{\mid x \in \mathbb{Z}}$ have the same behavior. In other words, knowing $\mathscr{B}_{n}$ over positive integers gives as much information as knowing $\mathscr{B}_{n}$ over the whole integers.

Theorem 3. If $N=N_{a} \times N_{b}, N_{a}$ and $N_{b}$ are primes, then $\mathscr{B}_{N}(x, y)_{\mid x \geq 4 N}=\left\langle P_{1}, P_{2}\right\rangle=\left\{P_{0}, P_{1}, P_{2}, P_{3}, P_{4}\right\}$ with $P_{3}=$ $P_{1}+P_{2}, \quad P_{4}=P_{2}+P_{3}=P_{1}+2 P_{2} \quad$ where $P_{1}=\left(\left(N_{a}+\right.\right.$ $\left.\left.N_{b}\right)^{2},\left(N_{a}^{2}-N_{b}^{2}\right)\right), P_{2}=\left(N_{b}\left(N_{a}+1\right)^{2}, N_{b}\left(N_{a}^{2}-1\right)\right), \quad P_{3}=$ $\left(N_{a}\left(N_{b}+1\right)^{2}, N_{a}\left(N_{b}^{2}-1\right)\right), P_{4}=\left((N+1)^{2}, N^{2}-1\right)$.

Proof. $\quad N=N_{a} \cdot N_{b}$, with $N_{a}$ and $N_{b}$ primes, then $\operatorname{Div}(N)=\left\{1, N_{a}, N_{b}, N\right\}$ and $|\operatorname{Div}(N)|=4$. By Proposition 6 and Theorem 2, $\operatorname{Card}\left(\mathscr{B}_{N}(x, y)_{\mid x \geq 4 N}\right)=U_{2}=5 \Rightarrow$
$\mathscr{B}_{N}(x, y)_{\mid x \geq 4 N}=\left\{P_{0}, P_{1}, P_{2}, P_{3}, P_{4}\right\}$. Furthermore, from Proposition 3, $\left(\left(N_{a}+N_{b}\right)^{2}, N_{a}^{2}-N_{b}^{2}\right), \quad\left(N_{b}\left(N_{a}+1\right)^{2}, N_{b}\right.$ $\left.\left(N_{a}^{2}-1\right)\right),\left(N_{a}\left(N_{b}+1\right)^{2}, N_{a}\left(N_{b}^{2}-1\right)\right),\left((N+1)^{2}, N^{2}-1\right)$ $\in \mathscr{B}_{N}(x, y)_{\mid x \geq 4 N}$. Considering the addition law on $\mathscr{B}_{N}(x, y)_{\mid x \geq 4 N}$ given by Proposition 1 and setting $P_{1}=\left(\left(N_{a}+N_{b}\right)^{2},\left(N_{a}^{2}-N_{b}^{2}\right)\right), \quad P_{2}=\left(N_{b}\left(N_{a}+1\right)^{2}, \quad N_{b}(\right.$ $\left.\left.N_{a}^{2}-1\right)\right), P_{3}=\left(N_{a}\left(N_{b}+1\right)^{2}, N_{a}\left(N_{b}^{2}-1\right)\right), P_{4}=\left((N+1)^{2}\right.$ ,$\left.N^{2}-1\right)$, one verifies as well in the polynomial ring $\mathrm{Q}\left[N_{a}, N_{b}, N\right]$ that $P_{1}+P_{2}=P_{3}, P_{2}+P_{3}=P_{1}+2 P_{2}=P_{4}$.

Thus, $\mathscr{B}_{N}(x, y)_{\mid x \geq 4 N}=\left\langle P_{1}, P_{2}\right\rangle$. It is not hard to see that $\left(\mathscr{B}_{N}(x, y)_{\mid x \geq 4 N},+, \cdot\right)$ is then a 2 -dimensional $\mathbb{Q}$-vector space with basis $\left\{P_{1}, P_{2}\right\}$.

\section{Application of $\mathscr{B}_{N}$ Parametrization to the Lattice Points on $x^{2}-y^{2}==N$ Fermat Equation}

In this section, we present results related to the lattice points on the arc of the hyperbola $x^{2}-y^{2}=N$ using results from $\mathscr{B}_{N}$ parametrization.

Theorem 4. $\forall(a, b) \in \mathscr{B}_{N}(x, y)_{\mid x \geq 4 N}$, if $\exists x \in \mathbb{Z}$ such that $a=4 x^{2}$, then $x$ verifies $x^{2}-y^{2}=N$. In this case, for positive lattice points, $\sqrt{N}$ is the lower bound for $x$.

Proof. Consider $X Y=N$, with $X=x+y$ and $Y=x-y$ which yields $x^{2}-y^{2}=N$.

$$
\begin{aligned}
(x+y-(x-y))^{2} & =(x+y)^{2}+(x-y)^{2}-2 N \\
& =\left[(x+y+x-y)^{2}-2 N\right]-2 N \\
& =(2 x)^{2}-4 N .
\end{aligned}
$$

Set $(2 x)^{2}=a$; then, $4 x^{2}=a$.

$a-4 N$ and $a$ are squares, yielding $a(a-4 N)$ to be also a square.

Then, there exists $b \in \mathbb{Z} / b^{2}=a(a-4 N)=a^{2}-4 N a=$ $\mathscr{B}_{N}(x, y)_{\mid x \geq 4 N}$. Hence, $\forall(a, b) \in \mathscr{B}_{N}(x, y)_{\mid x \geq 4 N}$, if $\exists x \in \mathbb{Z} / a=4 x^{2}, \Rightarrow \exists y \in \mathbb{Z} / x^{2}-y^{2}=N$. Also, as $a \geq 4 N$, then $4 x^{2} \geq 4 N$, considering positive $x, \Rightarrow x \geq \sqrt{N}$.

From now on, we denote $H_{N}=\left\{(x, y) \in \mathbb{Z}^{2} / x^{2}-y^{2}=\right.$ $N\}$ and $S$ as the algebraic set of $H_{N}$ over the integers.

Theorem 5. $\forall(x, y) \in \mathbb{Z}_{\geq 0}^{2}, \quad(x, y) \in H_{N}$; then, $(x, y) \in\left[\left[N^{1 / 2}\right\rceil,(1 / 2)(N+1)\right] \times[0,(1 / 2)(N-1)]$.

Proof. Assume $(x, y) \in \mathbb{Z}_{\geq 0}^{2}$ and $(x, y) \in H_{N}$.

From Theorem 1, $\forall(a, b) \in \mathscr{B}_{N}(x, y)_{\mid x \geq 4 N}, \quad(a, b) \in$ $[4 N, \quad N(N+2)+1] \times\left[0, N^{2}-1\right]$. From Theorem 4, $a=4 x^{2}$, where $(x, y)$ verifies $H_{N}$. If $a=4 N$, then $x^{2}=N$, $\Rightarrow x=N^{1 / 2}$ taking into account the assumption. Since we work over the integers, we take the ceiling for $x$. By the same, $y^{2}=x^{2}-N=0, \quad \Rightarrow y=0$. If $a=(N+1)^{2}$, then $x^{2}=(1 / 4)(N+1)^{2}, \Rightarrow x=(1 / 2)(N+1)$. By the same, $y^{2}=x^{2}-N=(1 / 4)(N+1)^{2}-N=(1 / 4)(N-1)^{2}, \quad \Rightarrow y=$ $(1 / 2)(N-1)$ taking into account the assumption. 


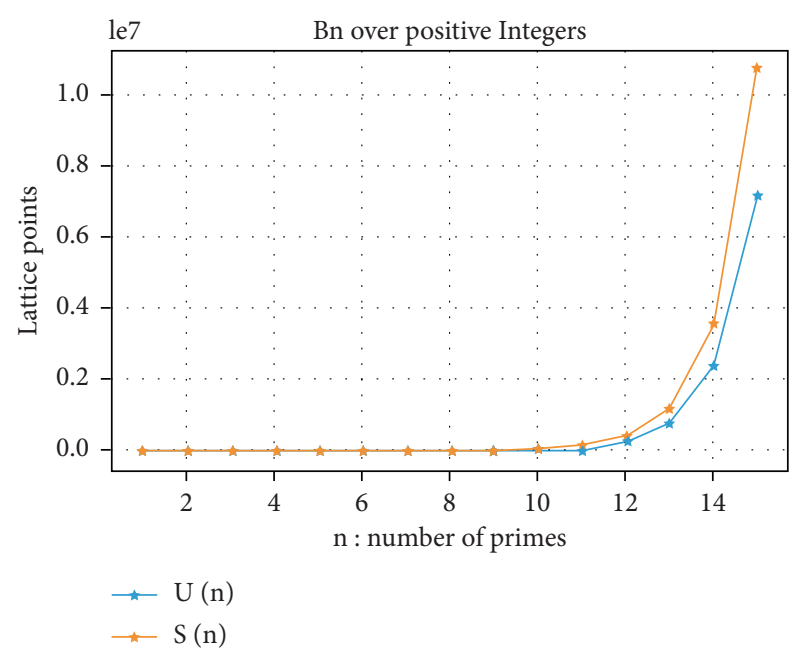

(a)

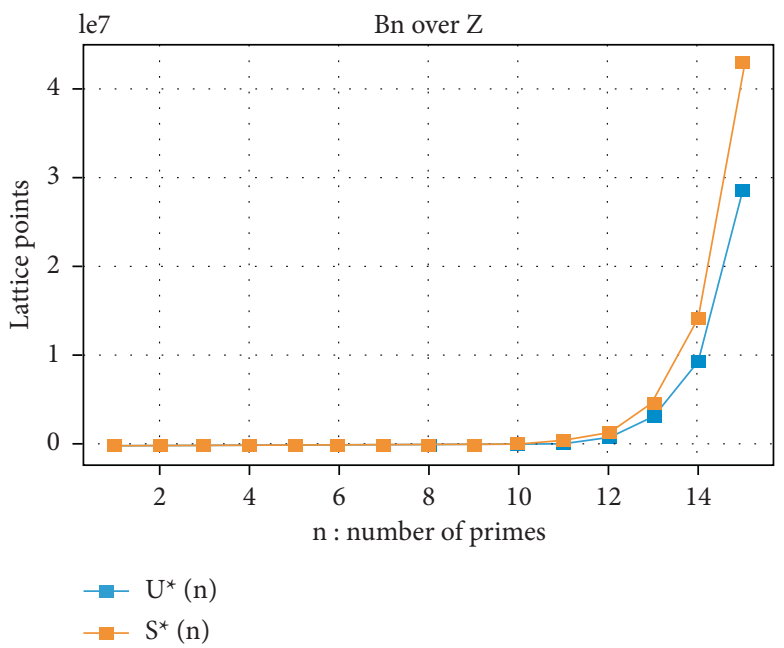

(b)

Figure 2: $\operatorname{Card}\left(\mathscr{B}_{n}(x, y)_{\mid x \geq 4 n}\right)$ (a) and $\operatorname{Card}\left(\mathscr{B}_{n}(x, y)_{\mid x \in \mathbb{Z}}\right)(\mathrm{b})$ over a sample of $n$ 's up to a product of 15 distinct primes.

TABLE 1: $U_{n}, S_{n} ; U_{n}^{*}, S_{n}^{*}$ based on a dataset of up to 15 distinct prime factors.

\begin{tabular}{lccccccccccccccc}
\hline$n$ & 1 & 2 & 3 & 4 & 5 & 6 & 7 & 8 & 9 & 10 & 11 & 12 & 13 & 14 & 15 \\
\hline$U_{n}$ & 2 & 5 & 14 & 41 & 122 & 365 & 1094 & 3281 & 9842 & 29525 & 88574 & 265721 & 797162 & 2391485 & 7174454 \\
$S_{n}$ & 2 & 7 & 21 & 62 & 184 & 549 & 1643 & 4924 & 14766 & 44291 & 132865 & 398586 & 1195748 & 3587233 & 10761687 \\
$U_{n}^{\star}$ & 8 & 20 & 56 & 164 & 488 & 1460 & 4376 & 13124 & 39368 & 118100 & 354296 & 1062884 & 3188648 & 9565940 & 28697816 \\
$S_{n}^{\star}$ & 8 & 28 & 84 & 248 & 736 & 2196 & 6572 & 19696 & 59064 & 177164 & 531460 & 1594344 & 4782992 & 14348932 & 43046748 \\
\hline
\end{tabular}

Hence, the bounds for $(x, y)$, $(x, y) \in\left[\left[N^{(1 / 2)}\right],(1 / 2)(N+1)\right] \times[0,(1 / 2)(N-1)]$.

Lemma 2. Given the sequence

$$
N_{k}= \begin{cases}6+4 k, & \text { if } k \geq 1, \\ 4, & \text { if } k=0\end{cases}
$$

Any term of this sequence and, respectively, any number of this form cannot be represented as difference of two squares.

Proof. If $k=0, N_{0}=N=4$, as $\operatorname{Div}(4)=\{1,2,4\}$, we have from Proposition 5, the following homomorphisms $\chi_{1 \rightarrow N}, \chi_{2 \rightarrow N}, \chi_{N \rightarrow N}$. From Theorem 2, (17), $\operatorname{Card}\left(\mathscr{B}_{4}(x, y)_{\mid x \geq 4 N}\right)=2+1=3$, and considering Proposition 5 , we have $\chi_{1 \rightarrow N} \longrightarrow(4 N, 0)=(16,0)$; $\chi_{2 \longrightarrow N}: \mathscr{B}_{2}(x, y) \longrightarrow \mathscr{B}_{N}(x, y),(9,3) \mapsto(2 \times 9,2 \times 3)=$ $(18,6) \quad$ where $(9,3) \in \mathscr{B}_{2}(x, y) \quad$ and $\quad \chi_{N \longrightarrow N} \longrightarrow$ $\left((N+1)^{2}, N^{2}-1\right)=(25,15) \Rightarrow \quad \mathscr{B}_{4}(x, y)_{\mid x \geq 16}=\{(16,0)$, $(18,6),(25,15)\}, \quad \nexists(a, b) \in \mathscr{B}_{4}(x, y)_{\mid x \geq 16} / a=4 x^{2}, x \in \mathbb{Z}$. Hence, 4 cannot be written as difference of two squares.

$\forall k>0, N_{k}=N=6+4 k=2(3+2 k)$, and $\operatorname{Div}(N)=$ $\{1,2,3+2 k\} \cup \operatorname{Div}(3+2 k) \backslash\{1,3+2 k\}$. By Proposition 3, since $2 \mid N$, then $(4 N+(N / 2), 3(N / 2))=(4 N+3+2 k$, $3(3+2 k)) \in \mathscr{B}_{N}(x, y)$. Also, as $3+2 k \mid N$, then $\left((3+2 k) N+2,2(3+2 k)^{2}-2\right) \in \mathscr{B}_{N}(x, y)$.

By induction, for $k=1, N_{1}=N=10, \operatorname{Div}(N)=$ $\{1,2,5,10\}$. From Theorem 2, Card $\left(\mathscr{B}_{n}(x, y)_{\mid x \geq 4 n}\right)=5$. We have from Proposition 5 the following homomorphisms: $\chi_{1 \longrightarrow N}, \chi_{2 \rightarrow N}, \chi_{5 \longrightarrow N}, \chi_{10 \longrightarrow N}$, and from Proposition 3, the following points: $1 \mid N \longrightarrow(4 N, 0)=(40,0) \in \mathscr{B}_{10}(x$, $y), 2 \mid N \longrightarrow(4 N+(N / 2), 3(N / 2))=(45,15) \in \mathscr{B}_{10}(x, y)$, $5 \mid N \longrightarrow(7 N+(N / 5), 24(N / 5))=(72,48) \in \mathscr{B}_{10}(x, y)$, $N \mid N \longrightarrow\left((N+2) N+1, N^{2}-1\right)=(121,99) \in \mathscr{B}_{10}(x, y)$, and since $2 \times 5=10$, then $\left((5+2)^{2}, 5^{2}-2^{2}\right)=(49,21) \epsilon$ $\mathscr{B}_{10}(x, y)$. Thus, $\mathscr{B}_{10}(x, y)_{\mid x \geq 40}=\{(40,0), \quad(45,15)$, $(49,21),(72,48),(121,99)\} . \quad \nexists(a, b) \in \mathscr{B}_{10}(x, y)_{\mid x \geq 16} / a=$ $4 x^{2}, x \in \mathbb{Z}$. Hence, 10 cannot be represented as difference of two squares.

For $k=2, N_{2}=N=14, \operatorname{Div}(N)=\{1,2,7,14\}$. From Theorem 2, Card $\left(\mathscr{B}_{N}(x, y)_{\mid x \geq 4 N}\right)=5$. We have from Proposition 5 the following homomorphisms: $\chi_{1 \rightarrow N}$, $\chi_{2 \longrightarrow N}, \chi_{7 \rightarrow N}, \chi_{14 \longrightarrow N}$, and from Proposition 3 , the following points: $\quad 1 \mid N \longrightarrow(4 N, 0)=(56,0) \in \mathscr{B}_{14}(x, y)$, $2 \mid N \longrightarrow(4 N+(N / 2), 3(N / 2))=(63,21) \in \mathscr{B}_{14}(x, y)$, $7 \mid N \longrightarrow\left(9 N+(N / 7),\left(7^{2}-1\right)(N / 7)\right)=(128,96) \in \mathscr{B}_{14}(x$, y), $N \mid N \longrightarrow\left((N+2) N+1, N^{2}-1\right)=(225,195) \in \mathscr{B}_{14}(x$, $y)$, and since $2 \times 7=14$, then $\left((7+2)^{2}, 7^{2}-2^{2}\right)=$ $(81,45) \in \mathscr{B}_{14}(x, y)$. Thus, $\mathscr{B}_{14}(x, y)_{\mid x \geq 56}=\{(56,0),(63,21)$, $(81,45),(128,96),(225,195)\} . \quad \nexists(a, b) \in \mathscr{B}_{14}(x, y)_{\mid x \geq 56} / a=$ $4 x^{2}, x \in \mathbb{Z}$. Hence, 14 cannot be represented as difference of two squares.

Assume the assumption to be true for $k$, i.e., for the term $N_{k}$, and let us show that it is true for $k+1$.

$$
\begin{aligned}
N_{k+1} & =N=6+4(k+1)=2(3+2(k+1)) \\
& =2(3+2 k)+2(2)=N_{k}+4 .
\end{aligned}
$$


Since $\quad N_{k}=6+4 k \Rightarrow N_{k+1}=6+4 k+4=6+4(k+1)$. Set $k^{\prime}=k+1$; then, $N_{k^{\prime}}=6+4 k^{\prime}$ (true by assumption).

Hence, $\nexists x \in \mathbb{Z} /\left(4 x^{2}, y\right) \in \mathscr{B}_{N_{k+1}}(x, y)_{\mid x \geq N_{k+1}}$.

Theorem 6. Consider the Fermat-Diophantine equation $x^{2}-y^{2}=N$. If $4 \mid(N-6)$, i.e., $(N-6) \equiv 0 \bmod 4$, then $S=\varnothing$.
Proof. This result is straightforward from Lemma 2.

Proposition 8. If $N$ is an odd prime, then $\operatorname{Card}\left(H_{N}\right)=4$. In this case,

$$
S=\left\{\begin{array}{l}
\left(\frac{1}{2}(N+1), \frac{1}{2}(N-1)\right),\left(-\frac{1}{2}(N+1),-\frac{1}{2}(N-1)\right) \\
\left(-\frac{1}{2}(N+1), \frac{1}{2}(N-1)\right),\left(\frac{1}{2}(N+1),-\frac{1}{2}(N-1)\right)
\end{array}\right\} .
$$

Proof. If $N$ is prime, from Theorem 2,

(1) $\operatorname{Card}\left(\mathscr{B}_{N}(x, y)_{\mid x \geq 4 N}\right)=2$.

(2) $\operatorname{Card}\left(\mathscr{B}_{N}(x, y)_{\mid \mathbb{Z}}\right)=4(2)-2=6$. From Proposition 5, through injective homomorphisms, we have $\mathscr{B}_{N}(x, y)_{\mid x \geq 4 N}=\left\{(4 N, 0),\left((N+1)^{2}, N^{2}-1\right)\right\}$.

Now since $N$ is an odd prime, $N+1$ is even, and $4 \mid(N+1)^{2}$. Setting $a=(N+1)^{2}, \quad \exists x \in \mathbb{Z} / a=4 x^{2} \Leftrightarrow x=$ $(1 / 2)(N+1)$ (we first consider the positive values of $x$ and $y)$, and as $x$ satisfies $x^{2}-y^{2}=N, \Rightarrow y=(1 / 2)(N-1)$. Then, over $\mathbb{Z}_{+}, H_{N}=\{(1 / 2)(N+1),(1 / 2)(N-1)\}$. Taking into account symmetric properties of $H_{N},(x, y) \in H_{N} \Rightarrow(-x, y)$, $(x,-y)$ and $(-x,-y) \in H_{N}$. Hence, $\operatorname{Card}\left(H_{N}\right)=4$ and $S=\left\{\begin{array}{c}((1 / 2)(N+1),(1 / 2)(N-1)),(-(1 / 2)(N+1),-(1 / 2)(N-1)), \\ (-(1 / 2)(N+1),(1 / 2)(N-1)),((1 / 2)(N+1),-(1 / 2)(N-1))\end{array}\right\}$.

Remark 2. If $N$ is an even prime, i.e., $N=2$, then Card $\left(H_{N}\right)=0$. This result is straightforward from Theorem 5 , since $4 \mid(N-6)$.

Proposition 9. If $N=N_{a}^{\alpha}, \quad N_{a}$ is prime. Then, $\operatorname{Card}\left(H_{N}\right)=2(\alpha+1)$. In this case,

$$
S=\left\{\begin{array}{c}
\left(\frac{1}{2}\left(N_{a}^{\alpha-i}+N_{a}^{i}\right), \frac{1}{2}\left(N_{a}^{\alpha-i}-N_{a}^{i}\right)\right),-\left(\frac{1}{2}\left(N_{a}^{\alpha-i}+N_{a}^{i}\right), \frac{1}{2}\left(N_{a}^{\alpha-i}-N_{a}^{i}\right)\right), \\
\left(\frac{1}{2}\left(N_{a}^{\alpha-i}+N_{a}^{i}\right),-\frac{1}{2}\left(N_{a}^{\alpha-i}-N_{a}^{i}\right)\right),\left(-\frac{1}{2}\left(N_{a}^{\alpha-i}+N_{a}^{i}\right),-\frac{1}{2}\left(N_{a}^{\alpha-i}-N_{a}^{i}\right)\right)
\end{array}\right\}
$$

Proof. If $N=N_{a}^{\alpha}, N_{a}$ is prime, from Theorem 2,

(1) $\operatorname{Card}\left(\mathscr{B}_{N}(x, y)_{\mid x \geq 4 N}\right)=\alpha+1$.

(2) $\operatorname{Card}\left(\mathscr{B}_{N}(x, y)_{\mid \mathbb{Z}}\right)=4 \alpha+2$. From Proposition 5, through injective homomorphisms, we have $\mathscr{B}_{N}(x, y)_{\mid x \geq 4 N}=\left\{(4 N, 0),\left(\left(N_{a}^{\alpha-i}+N_{a}^{i}\right)^{2}, N_{a}^{2(\alpha-i)}-\right.\right.$ $\left.\left.N_{a}^{2 i}\right),\left(N_{a}^{i}\left(N_{a}^{\alpha-i}+1\right)^{2}, N_{a}^{i}\left(N_{a}^{2(\alpha-i)}-1\right)\right)\right\}_{\mid i=0,1 \ldots \alpha}$. It is obvious to see that $N_{a}^{i}\left(N_{a}^{\alpha-i}+1\right)^{2}$ is not square $\forall i=\overline{0,1, \ldots, \alpha}$. Now since $N_{a}$ is prime, then $N_{a}^{\alpha-i}+$ $N_{a}^{i}$ is even $\forall i=\overline{0,1, \ldots, \alpha}, \Rightarrow\left(N_{a}^{\alpha-i}+N_{a}^{i}\right)^{2}$ is a multiple of 4 . From Theorem 4 , for this case, $\exists x \in \mathbb{Z}$ such that $a=\left(N_{a}^{\alpha-i}+N_{a}^{i}\right)^{2}=4 x^{2} \Rightarrow \mathscr{B}_{N}(x, y)_{\mid x \geq 4 N}$ has a total of $\alpha+1$ such terms since $i=\overline{0,1, \ldots, \alpha}$. Now considering the redundant terms each time $i=\alpha-i$, since $i$ ranges from 0 to $a$, then each term $N_{a}^{\alpha-i}+N_{a}^{i}$ is the same as $N_{a}^{i}+N_{\alpha}^{\alpha-i}$ because of the commutativity of the additive law. Then, we have exactly $\alpha+1 / 2$ such terms after removing the redundant terms for each of the $\alpha+1$ terms.

Then, over $\mathbb{Z}_{+}$, Card $\left(H_{N}\right)=(1 / 2)(\alpha+1)$. Taking into account the symmetry of $H_{N}, \quad(x, y) \in H_{N} \Rightarrow$ $(-x, y),(x,-y)$ and $(-x,-y) \in H_{N}$, then over $\mathbb{Z}$, we have $\operatorname{Card}\left(H_{N}\right)=4 \times(1 / 2)(\alpha+1)=2(\alpha+1)$.

Since $a=\left(N_{a}^{\alpha-i}+N_{a}^{i}\right)^{2}=4 x^{2}, \quad \Rightarrow x^{2}=(1 / 4)\left(N_{a}^{\alpha-i}+\right.$ $\left.N_{a}^{i}\right)^{2}$, then considering the positive values of $x$ and $y, x=$ $(1 / 2)\left(N_{a}^{\alpha-i}+N_{a}^{i}\right)$ and $y^{2}=x^{2}-N=(1 / 4)\left(N_{a}^{\alpha-i}+N_{a}^{i}\right)^{2}-$ $N_{a}^{\alpha}=(1 / 4) \quad\left(N_{a}^{2(\alpha-i)}+2 N_{a}^{\alpha}-4 N_{a}^{\alpha}+N_{a}^{2 i}\right)=(1 / 4)\left(N_{a}^{\alpha-i}-\right.$ $\left.N_{a}^{i}\right)^{2}, y=(1 / 2)\left(N_{a}^{\alpha-i}-N_{a}^{i}\right)$.

Hence

$S=$

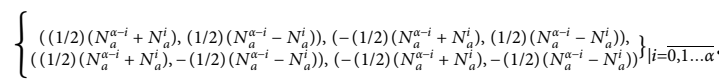

Proposition 10. Let $N=N_{a} \times N_{b}$, with $N_{a}, N_{b}$ odd primes. Then, $\operatorname{Card}\left(H_{N}\right)=8$. In this case, 


$$
S=\left\{\begin{array}{c}
\left(\frac{1}{2}\left(N_{a}+N_{b}\right), \frac{1}{2}\left(N_{a}-N_{b}\right)\right),\left(-\frac{1}{2}\left(N_{a}+N_{b}\right), \frac{1}{2}\left(N_{a}-N_{b}\right)\right), \\
\left(\frac{1}{2}\left(N_{a}+N_{b}\right),-\frac{1}{2}\left(N_{a}-N_{b}\right)\right),\left(-\frac{1}{2}\left(N_{a}+N_{b}\right),-\frac{1}{2}\left(N_{a}-N_{b}\right)\right), \\
\left(\frac{1}{2}(N+1), \frac{1}{2}(N-1)\right),\left(-\frac{1}{2}(N+1),-\frac{1}{2}(N-1)\right), \\
\left(-\frac{1}{2}(N+1), \frac{1}{2}(N-1)\right),\left(\frac{1}{2}(N+1),-\frac{1}{2}(N-1)\right)
\end{array}\right\} .
$$

Proof. Let $N=N_{a} \times N_{b}$ with $N_{a} N_{b}$ odd primes, from Theorem 2,

(1) $\operatorname{Card}\left(\mathscr{B}_{N}(x, y)_{\mid x \geq 4 N}\right)=5$.

(2) $\operatorname{Card}\left(\mathscr{B}_{N}(x, y)_{\mid \mathbb{Z}}\right)=4(5)-2=18$.

From Proposition 5, through injective homomorphisms, we have $\mathscr{B}_{N}(x, y)_{\mid x \geq 4 N}=$ $\left\{\begin{array}{c}(4 N, 0),\left(\left(N_{a}+N_{b}\right)^{2}, N_{a}^{2}-N_{b}^{2}\right),\left(N_{a}\left(N_{b}+1\right)^{2}, N_{a}\left(N_{b}^{2}-1\right),\right) \\ ,\left(N_{b}\left(N_{a}+1\right)^{2}, N_{b}\left(N_{a}^{2}-1\right),\right),\left((N+1)^{2}, N^{2}-1\right)\end{array}\right\}$.

Now since $N_{a}, N_{b}, N=N_{a} N_{b}$ are an odd, $N_{a}+N_{b}$ and $N+1$ are even. Then, $4 \mid\left(N_{a}+N_{b}\right)^{2}$ means 4 divides and $4 \mid(N+1)^{2}$. Set $a_{1}=\left(N_{a}+N_{b}\right)^{2}, \exists x \in \mathbb{Z} / a_{1}=4 x^{2} \Leftrightarrow x=$ $(1 / 2)\left(N_{a}+N_{b}\right)$, since $x$ satisfies $x^{2}-y^{2}=N, \Rightarrow y=(1 / 2)$ $\left(N_{a}-N_{b}\right) \quad$ and $\quad a_{2}=(N+1)^{2}, \quad \exists x \in \mathbb{Z} / a_{2}=4 x^{2} \Leftrightarrow x=$ $(1 / 2)(N+1)$ (we first consider the positive values of $x$ and $y)$, since $x$ satisfies $x^{2}-y^{2}=N, \Rightarrow y=(1 / 2)(N-1)$. Then, over $\mathbb{Z}_{+}, H_{N}=\left\{\left((1 / 2)\left(N_{a}+N_{b}\right),(1 / 2)\left(N_{a}-N_{b}\right)\right),((1 / 2)\right.$ $(N+1),(1 / 2)(N-1))\}$. Taking into account symmetric properties of $H_{N}, \quad(x, y) \in H_{N} \Rightarrow(-x, y),(x,-y)$ and $(-x,-y) \in H_{N}$. Hence, $\operatorname{Card}\left(H_{N}\right)=8$ and $S=$ $\int\left((1 / 2)\left(N_{a}+N_{b}\right),(1 / 2)\left(N_{a}-N_{b}\right)\right),\left(-(1 / 2)\left(N_{a}+N_{b}\right),(1 / 2)\left(N_{a}-N_{b}\right)\right)$, $\left\{\begin{array}{c}\left((1 / 2)\left(N_{a}+N_{b}\right),-(1 / 2)\left(N_{a}-N_{b}\right)\right),\left(-(1 / 2)\left(N_{a}+N_{b}\right),-(1 / 2)\left(N_{a}-N_{b}\right)\right) \\ ((1 / 2)(N+1),(1 / 2)(N-1)),(-(1 / 2)(N+1),-(1 / 2)(N-1),\end{array}\right\}$. $(-(1 / 2)(N+1),(1 / 2)(N-1)),((1 / 2)(N+1),-(1 / 2)(N-1))$

Proposition 11. Let $N=\prod_{i=1}^{n} p_{i}$ with, $p_{i}$ odd primes, then $\operatorname{Card}\left(H_{N}\right)=U_{n}=2 U_{n-1}=2^{n+1}$, and more generally, $\operatorname{Card}\left(H_{N}\right)=\left(1-\delta_{2 p_{i}}\right) 2^{n+1}$ for all distinct primes $p_{i}$, with $\delta_{2 p_{i}}$ being the Kronecker symbol which is given by

$$
\delta_{2 p_{i}}=\left\{\begin{array}{ll}
1, & \text { if } p_{i}=2 \\
0, & \text { else, }
\end{array} \forall i=\overline{1 \ldots n} .\right.
$$

Proof. We give a proof by induction.

For $n=1$, if $p_{1}=2, \operatorname{Card}\left(H_{N}\right)=0$ (true) from Remark 2 and if $p_{1} \neq 2$, Card $\left(H_{N}\right)=4$ (true) from Proposition 8 .

Now assume the proposition is true for $n$, and let us prove it to be also true for $n+1$.

For $n+1$, Card $\left(H_{N}\right)=U_{n+1}=2 U_{n}$. From the recurrence hypothesis, $U_{n}=2 U_{n-1}=\left(1-\delta_{2 p_{i}}\right) 2^{n+1}$; then, $\operatorname{Card}\left(H_{N}\right)=U_{n+1}=4 U_{n-1}=$

$2\left(1-\delta_{2 p_{i}}\right) 2^{n+1}=\left(1-\delta_{2 p_{i}}\right) 2^{n+2}$, hence the result.

Remark 3. If $N=N_{a} \times N_{b}, N_{a}, N_{b}$ primes Set $N_{a}=2$, then $S=\varnothing \quad$ and $\operatorname{Card}\left(H_{N}\right)=0$. Indeed, $N-6=2 N_{b}-6=2\left(N_{b}-3\right)$. Since $N_{b}$ is prime, $\Rightarrow N_{b}-3$ is even. Then, $\exists A \in \mathbb{Z}$ such that $N_{b}-3=2 A$, $\Rightarrow N-6=2(2 A)=4 A$. Since $4 \mid(N-6)$, From Theorem 5 , $S=\varnothing$.

Example 5. Solve $x^{2}-y^{2}=352706$ Here, $N=352706=2 \cdot 176353$. From Proposition 11, $\operatorname{Card}\left(H_{N}\right)=\left(1-\delta_{2 p_{i}}\right) 2^{n+1}$. Here, $\delta_{2 p_{i}}=1$ since $\exists p_{i}: p_{i}=2$. In this case, $\operatorname{Card}\left(H_{N}\right)=(1-1) 2^{2+P^{t}}=0$. This algebraic set is empty over the integers.

Proposition 12. Let $N=N_{a}^{\alpha} \times N_{b}^{\beta}$ with $N_{a}$ and $N_{b}$ odd primes Then, Card $\left(H_{N}\right)=2(\alpha+1)(\beta+1)$. In this case,

$$
S=\left\{\begin{array}{c}
\left(\frac{1}{2}\left(N_{a}^{\alpha-i} N_{b}^{\beta-j}+N_{a}^{i} N_{b}^{j}\right), \frac{1}{2}\left(N_{a}^{\alpha-i} N_{b}^{\beta-j}-N_{a}^{i} N_{b}^{j}\right)\right), \\
\left(\frac{1}{2}\left(N_{a}^{\alpha-i} N_{b}^{\beta-j}+N_{a}^{i} N_{b}^{j}\right)-\frac{1}{2}\left(N_{a}^{\alpha-i} N_{b}^{\beta-j}-N_{a}^{i} N_{b}^{j}\right)\right), \\
\left(\frac{1}{2}\left(N_{a}^{\alpha-i} N_{b}^{\beta-j}+N_{a}^{i} N_{b}^{j}\right),-\frac{1}{2}\left(N_{a}^{\alpha-i} N_{b}^{\beta-j}-N_{a}^{i} N_{b}^{j}\right)\right), \\
\left(-\frac{1}{2}\left(N_{a}^{\alpha-i} N_{b}^{\beta-j}+N_{a}^{i} N_{b}^{j}\right),-\frac{1}{2}\left(N_{a}^{\alpha-i} N_{b}^{\beta-j}-N_{a}^{i} N_{b}^{j}\right)\right)
\end{array}\right\}_{\mid i=0, \ldots, \alpha j=0, \ldots, \beta}
$$


Proof. From Proposition 5, through injective homomorphisms, we have $\mathscr{B}_{N}(x, y)_{\mid x \geq 4 N}=$ $\left\{\begin{array}{c}(4 N, 0),\left(\left(N_{a}^{\alpha-i} N_{b}^{\beta-j}+N_{a}^{i} N_{b}^{j}\right)^{2}, N_{a}^{2(\alpha-i)} N_{b}^{2(\beta-j)}-N_{a}^{2 i} N_{b}^{2 j}\right) \\ ,\left(N_{a}^{i} N_{b}^{j}\left(N_{a}^{\alpha-i} N_{b}^{\beta-j}+1\right)^{2}, N_{a}^{i} N_{b}^{j}\left(N_{a}^{2(\alpha-i)} N_{b}^{2(\beta-j)}-1\right)\right)\end{array}\right\}_{\mid i=0, \ldots, \alpha ; j=0, \ldots, \beta}$.

For each value of $i$, the value of $j$ covers $[0, \beta]$.

It is obvious to see that $N_{a}^{i} N_{b}^{j}\left(N_{a}^{\alpha-i} N_{b}^{\beta-j}+1\right)^{2}$ is not square $\forall i=\overline{0,1, \ldots, \alpha}$ and $j=\overline{0,1 \ldots \beta}$. Now since $N_{a}$ and $N_{b}$ are primes, then $N_{a}^{\alpha-i} N_{b}^{\beta-j}+N_{a}^{i} N_{b}^{j}$ is even $\forall i=\overline{0,1 \ldots \alpha}$ and $j=\overline{0,1 \ldots \beta}, \Rightarrow\left(N_{a}^{\alpha-i} N_{b}^{\beta-j}+N_{a}^{i} N_{b}^{j}\right)^{2}$ is a multiple of 4 . From Theorem 4, for this case, $\exists x \in \mathbb{Z}$ such that $a=\left(N_{a}^{\alpha-i} N_{b}^{\beta-j}+N_{a}^{i} N_{b}^{j}\right)^{2}=4 x^{2} . \Rightarrow \mathscr{B}_{N}(x, y)_{\mid x \geq 4 N}$ has a total of $(\alpha+1)(\beta+1)$ such terms since $i=\overline{0,1 \ldots \alpha}$ and $j=\overline{0,1 \ldots \beta}$. Now, considering the redundant terms each time $i=\alpha-i$ and $j=\beta-j$ then this leads each term $N_{a}^{\alpha-i} N_{b}^{\beta-j}+N_{a}^{i} N_{b}^{j}$ to be the same as $N_{a}^{i} N_{b}^{j}+N_{a}^{\alpha-i} N_{b}^{\beta-j}$, which is the same due to the commutativity of the common addition law, and then we have exactly $(1 / 2)(\alpha+1)(\beta+1)$ such terms without any redundancy, since all of the $(\alpha+$ 1) $(\beta+1)$ terms represent all terms together with their doublet.
Then, over $\mathbb{Z}_{+}$, $\operatorname{Card}\left(H_{N}\right)=(1 / 2)(\alpha+1)(\beta+1)$. Taking into account the symmetry of $H_{N}, \quad(x, y) \in H_{N} \Rightarrow$ $(-x, y),(x,-y)$ and $(-x,-y) \in H_{N}$, then over $\mathbb{Z}$, we have $\operatorname{Card}\left(H_{N}\right)=4 \times(1 / 2)(\alpha+1)(\beta+1)=2(\alpha+1)(\beta+1)$.

Since $a=\left(N_{a}^{\alpha-i} N_{b}^{\beta-j}+N_{a}^{i} N_{b}^{j}\right)^{2}=4 x^{2}, \quad \Rightarrow x^{2}=(1 / 4)$ $\left(N_{a}^{\alpha-i} N_{b}^{\beta-j}+N_{a}^{i} N_{b}^{j}\right)^{2}$; then, considering the positive values of $x$ and $y, \quad x=(1 / 2)\left(N_{a}^{\alpha-i} N_{b}^{\beta-j}+N_{a}^{i} N_{b}^{j}\right) \quad$ and $y^{2}=x^{2}-N=\quad(1 / 4)\left(N_{a}^{\alpha-i} N_{b}^{\beta-j}+N_{a}^{i} N_{b}^{j}\right)^{2}-N=$ $(1 / 4)\left(N_{a}^{\alpha-i} N_{b}^{\beta-j}+N_{a}^{i} N_{b}^{j}\right)^{2}-N_{a}^{\alpha} N_{b}^{\beta}=(1 / 4)\left(N_{a}^{2(\alpha-i)} N_{b}^{2(\beta-j)}+\right.$ $\left.N_{a}^{2 i} N_{b}^{2 j}-2 N_{a}^{\alpha} N_{b}^{\beta}\right)=(1 / 4) \quad\left(N_{a}^{\alpha-i} N_{b}^{\beta-j}-N_{a}^{i} N_{b}^{j}\right)^{2}$, $y=(1 / 2)\left(N_{a}^{\alpha-i} N_{b}^{\beta-j}-N_{a}^{i} N_{b}^{j}\right)$. Hence, $S=$ $\left.\left\{\begin{array}{l}\left((1 / 2)\left(N_{a}^{\alpha-i} N_{b}^{\beta-j}+N_{a}^{i} N_{b}^{j}\right),(1 / 2)\left(N_{a}^{\alpha-i} N_{b}^{\beta-j}-N_{a}^{i} N_{b}^{j}\right)\right), \\ \left((1 / 2)\left(N_{a}^{\alpha-i} N_{b}^{\beta-j}+N_{a}^{i} N_{b}^{j}\right),-(1 / 2)\left(N_{a}^{\alpha-i} N_{b}^{\beta-j}-N_{a}^{i} N_{b}^{j}\right)\right), \\ \left((1 / 2)\left(N_{a}^{\alpha-i} N_{b}^{\beta-j}+N_{a}^{i} N_{b}^{j}\right),-(1 / 2)\left(N_{a}^{\alpha-i} N_{b}^{\beta-j}-N_{a}^{i} N_{b}^{j}\right)\right), \\ \left(-(1 / 2)\left(N_{a}^{\alpha-i} N_{b}^{\beta-j}+N_{a}^{i} N_{b}^{j}\right),-(1 / 2)\left(N_{a}^{\alpha-i} N_{b}^{\beta-j}-N_{a}^{i} N_{b}^{j}\right)\right)\end{array}\right\}\right|_{\mid i=0, \ldots, \alpha ; j=0, \ldots, \beta}$

Proposition 13. If $N=\prod_{i=1}^{n} N_{i}^{\alpha_{i}}$, with $N_{i}$ odd primes. Then, $\operatorname{Card}\left(H_{N}\right)=2 \prod_{i=1}^{n}\left(\alpha_{i}+1\right)$. In this case,

$$
S=\left\{\left( \pm \frac{1}{2}\left(\prod_{i=1}^{j} N_{i}^{\alpha_{i}}+\prod_{i=j+1}^{n} N_{i}^{\alpha_{i}}\right), \pm \frac{1}{2}\left(\prod_{i=1}^{j} N_{i}^{\alpha_{i}}-\prod_{i=j+1}^{n} N_{i}^{\alpha_{i}}\right)\right)\right\}_{i \leq j \leq n} .
$$

Proof. We prove this by induction.

For $\quad n=1, \quad N=\prod_{i=1}^{1} N_{i}^{\alpha_{i}}=N_{1}^{\alpha_{1}}=2 \prod_{i=1}^{1}\left(\alpha_{i}+1\right)=$ $2\left(\alpha_{1}+1\right)$ which is true, since from Proposition 9, $\operatorname{Card}\left(H_{N}\right)=2\left(\alpha_{1}+1\right)$.

For $n=2, \quad N=\prod_{i=1}^{2} N_{i}^{\alpha_{i}}=N_{1}^{\alpha_{1}} N_{2}^{\alpha_{2}}=2 \prod_{i=1}^{2}\left(\alpha_{i}+1\right)=$ $2\left(\alpha_{1}+1\right)\left(\alpha_{2}+1\right)$ which is also true, since from Proposition 8 , $\operatorname{Card}\left(H_{N}\right)=2\left(\alpha_{1}+1\right)\left(\alpha_{2}+1\right)$.
Now assume the assumption to be true for $n$, and let us prove it to be true for $n+1$.

$N \cdot N_{n+1}^{\alpha_{n+1}}=\left(\prod_{i=1}^{n} N_{i}^{\alpha_{i}}\right) \cdot N_{n+1}^{\alpha_{n+1}}=\prod_{i=1}^{n+1} N_{i}^{\alpha_{i}}$, taking into account the assumption $\Rightarrow \operatorname{Card}\left(H_{N}\right)=2 \prod_{i=1}^{n+1}\left(\alpha_{i}+1\right)$.

From Proposition 5, through injective homomorphisms, we have

$$
\mathscr{B}_{N}(x, y)_{\mid x \geq 4 N}=\left\{\left(\left(\prod_{i=1}^{j} N_{i}^{\alpha_{i}}+\prod_{i=j+1}^{n} N_{i}^{\alpha_{i}}\right)^{2}, \prod_{i=1}^{j} N_{i}^{2 \alpha_{i}}-\prod_{i=j+1}^{n} N_{i}^{2 \alpha_{i}}\right),\left(\prod_{i=1}^{j} N_{i}^{\alpha_{i}}\left(\prod_{i=j+1}^{n} N_{i}^{\alpha_{i}}+1\right)^{2}, \prod_{i=1}^{j} N_{i}^{\alpha_{i}}\left(\prod_{i=j+1}^{n} N_{i}^{2 \alpha_{i}}-1\right)\right)\right\}
$$

It is obvious to see that $\prod_{i=1}^{j} N_{i}^{\alpha_{i}}\left(\prod_{i=1}^{n} N_{i}^{\alpha_{i}}+1\right)^{2}$ is not square $\forall i=\overline{1, \ldots n}$ and $j=\overline{1, \ldots n}$. Now since $N_{i}^{\alpha_{i}}$ is prime $\forall i=\overline{1, \ldots n}$, then $\prod_{i=1}^{j} N_{i}^{\alpha_{i}}+\prod_{i=j+1}^{n} N_{i}^{\alpha_{i}}$ is even and $\left(\prod_{i=1}^{j} N_{i}^{\alpha_{i}}+\prod_{i=j+1}^{n} N_{i}^{\alpha_{i}}\right)^{2}$ is a multiple of 4 . From Theorem 4 , for this case, $\exists x \in \mathbb{Z}$ such that $a=$ $\left(\prod_{i=1}^{j} N_{i}^{\alpha_{i}}+\prod_{i=j+1}^{n} N_{i}^{\alpha_{i}}\right)^{2}=4 x^{2} . \quad \Rightarrow x^{2}=(1 / 4)\left(\prod_{i=1}^{j} N_{i}^{\alpha_{i}}+\right.$ $\left.\prod_{i=j+1}^{n} N_{i}^{\alpha_{i}}\right)^{2}$; then, considering the positive values of $x$ and $y, \quad x=(1 / 2)\left(\prod_{i=1}^{j} N_{i}^{\alpha_{i}}+\prod_{i=j+1}^{n} N_{i}^{\alpha_{i}}\right)$ and $y^{2}=x^{2}-N=$ (1/4) $\left(\prod_{i=1}^{j} N_{i}^{\alpha_{i}}+\prod_{i=1}^{n} N_{i}^{\alpha_{i}}\right)^{2}-\prod_{i=1}^{n} N_{i}^{\alpha_{i}}$. Since $\prod_{i=1}^{n} N_{i}^{\alpha_{i}}=$ $\prod_{i=1}^{j} N_{i}^{\alpha_{i}}+\prod_{i=j+1}^{n} N_{i}^{\alpha_{i}}$, then

$$
y^{2}=\frac{1}{4}\left(\prod_{i=1}^{j} N_{i}^{2 \alpha_{i}}+\prod_{i=j+1}^{n} N_{i}^{2 \alpha_{i}}+2 \prod_{i=1}^{j} N_{i}^{\alpha_{i}} \prod_{i=j+1}^{n} N_{i}^{\alpha_{i}}-4 \prod_{i=1}^{j} N_{i}^{\alpha_{i}} \prod_{i=j+1}^{n} N_{i}^{\alpha_{i}}\right)=\frac{1}{4}\left(\prod_{i=1}^{j} N_{i}^{\alpha_{i}}-\prod_{i=j+1}^{n} N_{i}^{\alpha_{i}}\right)^{2} .
$$


$y=(1 / 2)\left(\prod_{i=1}^{j} N_{i}^{\alpha_{i}}-\prod_{i=j+1}^{n} N_{i}^{\alpha_{i}}\right)$. Taking into account the symmetry of $H_{N},(x, y) \in H_{N} \Rightarrow(-x, y),(x,-y)$ and $(-x,-y) \in H_{N}$; then, over $\mathbb{Z}$, we have

$$
S=\left\{\left( \pm \frac{1}{2}\left(\prod_{i=1}^{j} N_{i}^{\alpha_{i}}+\prod_{i=j+1}^{n} N_{i}^{\alpha_{i}}\right), \pm \frac{1}{2}\left(\prod_{i=1}^{j} N_{i}^{\alpha_{i}}-\prod_{i=j+1}^{n} N_{i}^{\alpha_{i}}\right)\right)\right\}_{i \leq j \leq n} .
$$

\section{Discussion}

We have exposed the forms of the Fermat equation $x^{2}-y^{2}=N$, dependently on the different forms of $N$, for which we have proved the cardinal over the integers to be 0 , 4 , and 8 , of the form $2 \prod_{i=1}^{n}\left(\alpha_{i}+1\right)$ or $\left(1-\delta_{2 p_{i}}\right) 2^{n+1}$.

Over $\mathbb{Z}_{>0}, x^{2}-y^{2}=N$ has only one nontrivial solution for a RSA modulus $N$.

Proposition 14. $\forall(x, y) \in\left[\left\lceil N^{1 / 2}\right],(1 / 2)(N+1)\right] \times[0$, $(1 / 2) \quad(N-1)], \quad(x, y) \in H_{N}$ with the probability $P=\prod_{i=1}^{n}\left(\alpha_{i}+1\right) / N+1-2\left\lceil N^{1 / 2}\right\rceil$.

Proof. Set $N=\prod_{i=1}^{n} N_{i}^{\alpha_{i}}$, with $N_{i}$ odd primes. From Proposition 13 , over $\mathbb{Z}_{\geq 0}$, Card $\left(H_{N}\right)=(1 / 2) \prod_{i=1}^{n}\left(\alpha_{i}+1\right)$ and from Theorem 5, the length of the $x$ interval is $l=N+1 / 2-$ $\left\lceil N^{1 / 2}\right\rceil=N+1-2\lceil\sqrt{N}\rceil / 2 . \quad \Rightarrow P=\left(\operatorname{Card}\left(H_{N}\right)_{\mid \mathbb{Z} \geq 0} / l\right)=$ $\left((1 / 2) \prod_{i=1}^{n}\left(\alpha_{i}+1\right) / N+1-2\lceil\sqrt{N}\rceil / 2\right)=\left(\prod_{i=1}^{n}\left(\alpha_{i}+1\right) / N+\right.$ $1-2\lceil\sqrt{N}\rceil)$.

\section{Conclusion}

In this paper, we have presented algebraic results on lattice points of the arc on the conics $x^{2}-d y^{2}=N$ for $d=1$, which is the Fermat factorization equation for which cardinals, forms of the algebraic set and exact upper and lower bounds are given using a particular hyperbola parametrization. These results provide further information on the structure of the algebraic set of this equation by exposing particularly the following.

(i) The general forms of lattice points.

(ii) The cardinals and the exact number of solutions.

(iii) The distribution of its lattice points over the integers.

As a future work, we shall apply these results in the square sieving methods of factorization (mainly the quadratic sieve) and evaluate any resulting impact and performance.

\section{Data Availability}

The algorithms were developed in Python, and the source codes are available from the corresponding author upon request.

\section{Conflicts of Interest}

The authors declare that there are no conflicts of interest regarding the publication of this paper.

\section{References}

[1] J. Cilleruelo and J. Jiménez-Urroz, "Lattice points on hyperbolas," Journal of Number Theory, vol. 63, no. 2, pp. 267-274, 1997.

[2] J. Cilleruelo and J. Jimenez-Urroz, "The Hyperbola $x y=N$," Journal de Théorie des Nombres de Bordeaux, Tome, vol. 12, no. 1, pp. 87-92, 2000.

[3] J. Cilleruelo and A. Cordoba, "Trigonometric polynomials and lattice points," Proceeding of the American Mathematical Society, vol. 115, no. 4, 1992.

[4] J. John Brillhart, R. Blecksmith, and M. Decaro, "Using conic sections to factor integers," The American Mathematical Monthly, vol. 123, no. 2, pp. 168-174, 2016.

[5] B. Emanuele, M. Nadir, J. Antonio, and E. Michele, "Pointgroups over singular cubics," 2020, https://arxiv.org/pdf/2001. 04288.pdf.

[6] R. Taton, "L' essay pour les coniques de pascal," Revue d'Histoire des Sciences et de Leurs Applications, vol. 8, no. 1, pp. 1-18, 1955.

[7] A. K. Lenstra, H. W. Lenstra, M. Manasse, and J. M. Pollard, "Carl pomerance the number field sieve," Proceedings of Symposia in Applied Mathematics, vol. 48, 1994.

[8] C. Pomerance, "The quadratic sieve factoring algorithm," in Proceedings of the Advances in Cryptology, Proceeding of EUROCRYPT'84', LNCS 209, pp. 169-182, Springer-Verlag, Berlin, Germany, 1985.

[9] Fermat, Oeuvres de Fermat, vol. 2, p. 256, 1894.

[10] J. McKee, "Speeding Fermat's factoring method," Mathematics of Computation, vol. 68, no. 228, pp. 1729-1737, 1999.

[11] R. L. Rivest, A. Shamir, and L. Adleman, "A method for obtaining digital signatures and public-key cryptosystems," Communications of the ACM, vol. 21, no. 2, pp. 120-126, 1978.

[12] H. Jin, K. Jeong, and J.-H. Kwon, "Integral points on hyperbolas," Journal of the Korean Mathematical Society, vol. 34, no. 1, pp. 149-157, 1997.

[13] K. Zelator, "Integral points on hyperbolas over Z: a special case," 2009, https://arxiv.org/abs/0907.3675.

[14] Y. Kim, "On some Behavior of integral points on a Hyperbola," Bulletin of the Korean Mathematical Society, vol. 50, no. 4, pp. 1243-1259, 2013.

[15] B. Emanuele, N. Murru, J. Antonio, Di Scala, and E. Michele, "Group law on affine conics and applications to cryptography," Journal of Applied Mathematics and Computation, Elsevier, 2020.

[16] G. R. Bansimba, R. F. Babindamana, and B. G. R. Bossoto, "Some arithmetical properties on hyperbola," JP Journal of Algebra, Number Theory and Applications, vol. 50, no. 1, pp. 45-100, 2021. 